\title{
Autoantibodies linked to autoimmune diseases associate with COVID-19 outcomes
}

Gabriela Crispim Baiocchi ${ }^{1, *}$, Aristo Vojdani ${ }^{2,3, *}$, Avi Z Rosenberg ${ }^{4}$, Elroy Vojdani ${ }^{5}$, Gilad Halpert ${ }^{6,7,8}$, Yuri Ostrinski $^{6,7,8}$, Israel Zyskind ${ }^{9,10}$, Igor Salerno Filgueiras ${ }^{1}$, Lena F. Schimke ${ }^{1}$, Alexandre H. C. Marques ${ }^{1}$, Lasse M. Giil $^{11}$, Yael Bublil Lavi ${ }^{12}$, Jonathan I. Silverberg ${ }^{13}$, Jason Zimmerman ${ }^{10}$, Dana Ashley Hill ${ }^{14}$, Amanda Thornton ${ }^{14}$, Myungjin Kim ${ }^{15}$, Roberta De Vito ${ }^{16}$, Dennyson Leandro M. Fonseca ${ }^{17}$, Desireé Rodrigues Plaça ${ }^{17}$, Paula Paccielli Freire $^{1}$, Niels Olsen Saraiva Camara ${ }^{1}$, Vera Lúcia Garcia Calich ${ }^{1}$, Harald Heidecke ${ }^{18}$, Miriam T. Lattin ${ }^{19}$, Hans D. $\mathrm{Ochs}^{20}$, Gabriela Riemekasten ${ }^{21}$, Howard Amital ${ }^{22,6,23,7}$, Otavio Cabral-Marques ${ }^{1,17,24 *}$, Yehuda Shoenfeld, ${ }^{7,6,8, *}$

${ }^{1}$ Department of Immunology, Institute of Biomedical Sciences, University of São Paulo, São Paulo, SP, Brazil.

${ }^{2}$ Department of Immunology, Immunosciences Laboratory, Inc., Los Angeles, CA, United States.

${ }^{3}$ Cyrex Laboratories, LLC 2602 S. 24th St., Phoenix, AZ 85034 USA

${ }^{4}$ Department of Pathology, Johns Hopkins University, Baltimore, Maryland, USA

${ }^{5}$ Regenera Medical 11860 Wilshire Blvd., Ste. 301, Los Angeles, CA 90025 USA

${ }^{6}$ Ariel University, Israel

${ }^{7}$ Zabludowicz Center for Autoimmune Diseases, Sheba Medical Center, , Tel-Hashomer, Israel

${ }^{8}$ Saint Petersburg State University Russia

${ }^{9}$ Department of Pediatrics, NYU Langone Medical Center, New York, NY, USA

${ }^{10}$ Maimonides Medical Center, Brooklyn, NY, USA

${ }^{11}$ Department of Internal Medicine, Haraldsplass Deaconess Hospital, Bergen, Norway

${ }^{12}$ Department of Chemistry Ben Gurion University Beer-Sheva, 84105, Israel

${ }^{13}$ Department of Dermatology, George Washington University School of Medicine and Health Sciences, Washington, DC, USA

${ }^{14}$ ResourcePath, Sterling, VA

${ }^{15}$ Data Science Initiative at Brown University, Providence, RI, USA

${ }^{16}$ Department of Biostatistics and the Data Science Initiative at Brown University, Providence, RI, USA

${ }^{17}$ Department of Clinical and Toxicological Analyses, School of Pharmaceutical Sciences, University of São Paulo, São Paulo, SP, Brazil

${ }^{18}$ CellTrend Gesellschaft mit beschränkter Haftung $(\mathrm{GmbH})$, Luckenwalde, Germany

${ }^{19}$ Department of Biology, Yeshiva University, Manhatten, NY, USA

${ }^{20}$ Department of Pediatrics, University of Washington School of Medicine, and Seattle Children's Research Institute, Seattle, WA, USA

${ }^{21}$ Department of Rheumatology, University Medical Center Schleswig-Holstein Campus Lübeck, Lübeck, Germany

${ }^{22}$ Department of Medicine B, Sheba Medical Center, Tel Hashomer, Israel

${ }^{23}$ Sackler Faculty of Medicine, Tel-Aviv University, Tel-Aviv, Israel

${ }^{24}$ Network of Immunity in Infection, Malignancy, and Autoimmunity (NIIMA), Universal Scientific Education and Research Network (USERN), São Paulo, SP, Brazil

*Contributed

equally

\section{Corresponding authors:}

Yehuda Shoenfeld, MD, FRCP, MaACR

Zabludowicz Center for Autoimmune Diseases,

Sheba Medical Center, affiliated with the Sackler

Faculty of Medicine, Tel-Aviv University

Tel-Hashomer 5265601, Israel

Email: shoenfel@post.tau.ac.il

Tel: $\quad 972-3-5308070$

Fax: $\quad 972-3-5352855$

\section{Gabriela Crispim Baiocchi}

Department of Immunology

Institute of Biomedical Sciences

University of São Paulo

Lineu Prestes Avenue, 1730, São Paulo, SP, 05508-900, Brazil

Email: gabrielacbaiocchi@icb.usp.br

Phone: +55 11990005454
Otavio Cabral-Marques, $\mathrm{MSc}, \mathrm{PhD}$

Department of Immunology

Institute of Biomedical Sciences

University of São Paulo

Lineu Prestes Avenue, 1730, São Paulo, SP, 05508-

900, Brazil

Email: otavio.cmarques@usp.br

Phone: +55 1197464202

Fax:+551130917397 


\section{ABSTRACT}

2 The SARS-CoV-2 infection is associated with increased levels of autoantibodies targeting 3 immunological proteins such as cytokines and chemokines. Reports further indicate that COVID-

419 patients may develop a wide spectrum of autoimmune diseases due to reasons not fully 5 understood. Even so, the landscape of autoantibodies induced by SARS-CoV-2 infection remains 6 uncharted territory. To gain more insight, we carried out a comprehensive assessment of 7 autoantibodies known to be linked to diverse autoimmune diseases observed in COVID-19 8 patients, in a cohort of 248 individuals, of which171 were COVID-19 patients (74 with mild, 65 9 moderate, and 32 with severe disease) and 77were healthy controls. Dysregulated autoantibody 10 serum levels, characterized mainly by elevated concentrations, occurred mostly in patients with 11 moderate or severe COVID-19 infection, and was accompanied by a progressive disruption of 12 physiologic $\operatorname{IgG}$ and $\operatorname{IgA}$ autoantibody signatures. A similar perturbation was found in patients 13 with anosmia. Notably, autoantibody levels often accompanied anti-SARS-CoV-2 antibody 14 concentrations, being both indicated by random forest classification as strong predictors of 15 COVID-19 outcome, together with age. Moreover, higher levels of autoantibodies (mainly IgGs)

16 were seen in the elderly with severe disease compared with young COVID-19 patients with 17 severe disease. These findings suggest that the SARS-CoV-2 infection induces a broader loss of 18 self-tolerance than previously thought, providing new ideas for therapeutic interventions. outcomes 


\section{Introduction}

25 Since the first case in December 2019, more than 395 million confirmed cases of the

26 Coronavirus disease 2019 (COVID-19), including 5,7 million deaths, were reported ${ }^{1}$. COVID-19

27 is caused by the severe acute respiratory syndrome coronavirus 2 (SARS-CoV-2), a single-

28 stranded RNA virus that belongs to the Coronaviridae family ${ }^{2}$. Subsequently, new SARS-CoV-2

29 variants have kept on emerging, each presenting with selective advantages ${ }^{3-5}$ and threatening the

30 possibility to dominate the COVID-19 pandemic $^{6}$. Concurrently, a massive scientific endeavor to

31 understand COVID-19 pathophysiology has found evidence that patients with severe COVID-19

32 may present with systemic immune dysregulation of both the innate and adaptive immune

33 responses. This manifests mainly as cytokine storm syndrome ${ }^{7}$, hyperactivation of both myeloid

34 and lymphoid cells ${ }^{7,8}$, characterized by neutrophilia and lymphopenia) ${ }^{9}$. . In addition, recent

35 reports from our group and others ${ }^{10,11,12}$ indicate that patients with COVID-19 have high levels of

36 autoantibody production, suggesting that SARS-CoV-2 can trigger features resembling systemic

37 autoimmune disease ${ }^{13-17}$.

In healthy individuals, autoantibodies are present at physiological levels regulating a

39 myriad of physiological functions, which are essential to maintain body homeostasis ${ }^{18-21}$.

40 However, abnormal levels of autoantibodies often predict preclinical autoimmune diseases ${ }^{22,23}$ as

41 well as promote or exacerbate inflammatory conditions by triggering or exacerbating

42 inflammation, dysregulating biological pathways, and inducing cell lysis ${ }^{24}$. Therefore,

43 autoantibody levels in COVID-19 may have clinical implications. The identified link between

44 SARS-CoV-2 and altered autoantibody profiles suggest that similar to other viruses ${ }^{25-28}$, SARS-

45 CoV-2 infection may result in a life-threatening generalized loss of self-tolerance ${ }^{13-15,29}$. This

46 includes antiphospholipid syndrome ${ }^{30}$, Gillian-Barré syndrome ${ }^{31}$, polyneuritis cranialis ${ }^{32}$, new- 
47 onset type 1 diabetes ${ }^{33}$, autoimmune hemolytic anemia ${ }^{34}$, Graves' disease ${ }^{35}$, immune 48 thrombocytopenic purpura ${ }^{36}$, $\operatorname{arthritis}^{37}$, and systemic lupus erythematosus ${ }^{16,38}$. However, the full

49 spectrum of autoantibodies observed in COVID-19 patients which could indicate a broad loss of

50 self-tolerance, has not been fully characterized. Among the recently reported autoantibodies

51 identified in COVID-19 are those targeting immune related proteins such as type I interferons

52 (IFNs) ${ }^{11}$, the exoproteome (cytokine, chemokine, and their receptors as well as complement

53 factors) ${ }^{10}, \mathrm{G}$ protein-coupled receptors (GPCRs) and renin-angiotensin system (RAS)-related

54 molecules ${ }^{12}$.

While a select group of autoantibodies observed in classical autoimmune diseases have

56 been associated with COVID-19 severity such as anti-nuclear antibodies (ANAs ${ }^{39}$ ), ribosomal P

57 proteins, chromatin proteins, and thyroid antigens ${ }^{40}$ although comprehensive studies broadly

58 characterizing autoantibodies induced by SARS-CoV-2 are lacking. Furthermore, high levels of

59 antiphospholipid autoantibodies were linked to severe COVID-19 by inducing neutrophil

60 extracellular traps (NETs) and venous thrombosis ${ }^{16,41-44}$. Based on these observations,

61 autoantibodies associated with diverse autoimmune diseases and the loss of self-tolerance might

62 be associated with the severity of COVID-19 as previously indicated ${ }^{14,16,17,40,45,46}$ and by

63 extension suggest that loss of self-tolerance is a feature of severe COVID-19. To better

64 characterize the immunopathology of COVID-19 it is imperative to determine the landscape of

65 autoantibodies associated with the severity of SARS-CoV-2 infection, which will lead to

66 improve therapeutic interventions. To achieve this goal, we have characterized the spectrum of

67 autoantibodies linked to autoimmune diseases in patients with different COVID-19 clinical

68 outcomes, using a systems immunology approach. 


\section{METHODS}

\section{Study Participants}

72 A total of 248 adults belonging to Jewish communities from 5 states of the United States of

73 America were enrolled in this study. Of these, 171 patients had COVID-19 symptoms

74 (Supplementary Table 1) and a positive SARS-CoV-2 test by nasopharyngeal swab and PCR

75 analysis (polymerase chain reaction) before receiving any SARS-CoV-2 vaccine. These patients

76 participated in an online survey developed to determine the most common symptoms and

77 outcomes of SARS-CoV-2 infection ${ }^{47,48}$. The SARS-CoV-2 positive cohort was classified as

78 mild COVID-19 ( $\mathrm{n}=74$; fever duration $\leq 1$ day; peak temperature of $37.8 \mathrm{C}$ ), moderate COVID-

$7919(\mathrm{n}=63$; fever duration $\geq 7$ days; peak fever of $\geq 38.8 \mathrm{C})$, and severe COVID-19 groups $(\mathrm{n}=32$;

80 severe symptoms and requiring supplemental oxygen therapy) according to the World Health

81 Organization (WHO) severity classification ${ }^{49}$. Details of the survey study, patient demographics,

82 and symptoms were previously described ${ }^{47,48}$ and can be found in Supplementary Table 1. We

83 also included 77 randomly selected age- and sex-matched healthy controls from the same Jewish

84 population who were SARS-CoV-2 negative and did not present any COVID-19 symptoms. All

85 healthy controls and all patients provided informed written consent to participate in the study,

86 which was performed in accordance with the Declaration of Helsinki. The study was approved

87 by the IntegReview institutional review board (Coronavirus Antibody Prevalence Study, CAPS-

88 613). In addition, this study followed the reporting guidelines of Strengthening the Reporting of

89 Observational Studies in Epidemiology (STROBE).

90

91 Measurements of anti-SARS-CoV-2 antibody and autoantibodies linked to autoimmune

92 diseases 
All collected sera were tested for IgG anti-SARS-CoV-2 antibody using the ZEUS

94 SARS-CoV-2 ELISA Test System according to the manufacturer's instructions (ZEUS

95 Scientific, New Jersey, USA). This is an ELISA test that measures IgG antibodies to spike and

96 nucleoprotein combined. Serum IgG autoantibodies against nuclear antigen (ANA), extractable

97 nuclear antigen (ENA), double-stranded DNA (dsDNA), actin, mitochondrial M2, and

98 rheumatoid factor (RF) were measured using commercial ELISA kits obtained from INOVA

99 Diagnostics (San Diego, CA, USA). In addition, IgG and IgA antibodies against 52 different

100 autoantigens ${ }^{50-58}$ were measured by in-house ELISA procedure at Immunosciences Lab., Inc.

101 (Los Angeles, CA USA) in blinded fashion. See Supplementary Table 2 for the autoantigens

102 tested.. For the measurement of these antibodies, one hundred $\mathrm{mL}$ of each autoantigen at the

103 optimal concentration, prepared in $0.01 \mathrm{M}$ PBS pH 7.4 were added to a series of microtiter

104 plates. A set of plate wells were also coated with $2 \%$ bovine serum albumin (BSA) or human

105 serum albumin (HSA) and used as controls. The ELISA plates were incubated overnight at $4^{\circ} \mathrm{C}$

106 and then were washed five times with $250 \mathrm{ml}$ of $0.01 \mathrm{M}$ PBS containing $0.05 \%$ Tween $20 \mathrm{pH}$

107 7.4. The non-specific binding of immunoglobins was prevented by the addition of $200 \mathrm{~mL}$ of $2 \%$

108 BSA in PBS, which was then incubated overnight at $4^{\circ} \mathrm{C}$. Plates were washed and the serum

109 samples from healthy controls and the SARS-CoV-2 patients were diluted at 1:50 for the

110 determination of $\operatorname{IgA}$ antibody, and 1:100 for the determination of $\operatorname{IgG}$ antibody in serum diluent

111 buffer or $1 \%$ BSA in PBS containing $0.05 \%$ Tween 20 was added to the wells of ELISA plates,

112 which were then incubated for one hour at room temperature. Plates were washed, followed by

113 the addition of alkaline phosphatase goat anti-human $\operatorname{IgG}$ or $\operatorname{IgA} \mathrm{F}(\mathrm{ab})$,2 fragments (KPI,

114 Gaithesburg, MD, USA) at an optimal dilution of 1:200 for IgA and 1:600 for IgG in 1\% BSA-

115 PBS. The plates were then incubated for another hour at room temperature. After five washings 
116 with PBS-Tween buffer, the enzyme reaction was started by adding $100 \mathrm{~mL}$ of para-

117 nitrophenylphosphate in $0.1 \mathrm{~mL}$ diethanolamine buffer $1 \mathrm{mg} / \mathrm{mL}$ containing $1 \mathrm{mM} \mathrm{MgCl} \mathrm{M}_{2}$ and

118 sodium azide $\mathrm{pH}$ 9.8. Forty-five minutes later, the reaction was stopped with $50 \mathrm{~mL}$ of $1 \mathrm{~N}$

$119 \mathrm{NaOH}$. The optical density (OD) was read at $405 \mathrm{~nm}$ with a microtiter plate reader. The ODs of

120 the control wells containing only HSA or BSA which were less than 0.15 were subtracted from

121 all other wells to exclude non-specific binding. The ELISA index for each antibody was

122 calculated and the background of the ELISA assay was not more than 0.150 O.D.

\section{Multistudy factor analysis}

125 Multistudy factor analysis (MSFA) was performed using the R package called MSFA ${ }^{59}$, which 126 uses either a fast expectation conditional-maximization algorithm and a Gibbs Sampling

127 algorithm $^{60}$ for the parameter estimate. Here, we adopt the Gibbs Sampling algorithm to estimate 128 the parameter in a Bayesian framework ${ }^{60}$. MSFA is an unsupervised and inferential analysis used 129 to identify common and study-specific factors shared by the control and COVID-19 groups as 130 previously described $^{61}$.

\section{Differences in autoantibody levels}

133 Box plots showing differences in autoantibodies from COVID-19 patient groups (mild, moderate 134 and severe) and healthy controls were generated using the R version 4.0.5 (The R Project for 135 Statistical Computing. https://www.r-project.org/), R studio Version 1.4.1106 (R-Studio. 136 https://www.rstudio.com/), and the $\mathrm{R}$ packages ggpubr, lemon, and ggplot2. Statistical 137 differences in autoantibody levels were assessed using a two-sided Wilcoxon rank-sum ${ }^{10}$. 
Principal component analysis (PCA)

140 PCA with spectral decomposition ${ }^{62,63}$ was carried out to measure the stratification power of the 141 autoantibodies in distinguishing COVID-19 (mild, moderate, and severe patients) and healthy 142 controls. PCA was performed using the $\mathrm{R}$ functions prcomp and princomp, through the 143 factoextra package (PCA in R: prcomp vs princomp $)^{64}$.

\section{Machine learning model and autoantibody ranking}

146 We employed random forest model to construct a classifier for discriminating controls and mild, 147 moderate, and severe COVID-19 patients. The aim of this approach was to identify the most 148 significant predictors for severe COVID-19. We trained the random forest model using the 149 functionalities of the R package randomForest (version 4.6.14) ${ }^{65}$. Five thousand trees were used, 150 and 3 variables were resampled. A follow-up analysis was conducted with the Gini decrease, the 151 number of nodes and mean minimum depth as criteria to determine variable importance. The 152 adequacy of the random forest model as a classifier was assessed through the out-of-bags error 153 rate and the ROC curve. For cross-validation, we split the dataset into training and testing sets, 154 using $75 \%$ of the observations for training and $25 \%$ for testing.

\section{Autoantibody correlation signatures}

157 Bivariate correlation analyses of autoantibodies for each group (controls and mild, moderate and 158 severe COVID-19 patients) were performed using the corrgram, psych and inlmisc R packages. 159 In addition, circle plots of autoantibody correlation were built using the $\mathrm{R}$ packages qgraph, 
medRxiv preprint doi: https://doi.org/10.1101/2022.02.17.22271057; this version posted February 18, 2022. The copyright holder for this preprint (which was not certified by peer review) is the author/funder, who has granted medRxiv a license to display the preprint in perpetuity.

It is made available under a CC-BY-NC-ND 4.0 International license .

160 ggplot2, psych, inlmisc to visualize the patterns of Spearman's rank correlation coefficients

161 between autoantibodies.

162

163 RESULTS

164 Progressive increase of autoantibodies in COVID-19 patients according to disease severity

165 Here we investigated the serum levels of $\operatorname{IgG}$ and $\operatorname{IgA}$ autoantibodies targeting 58 and 52

166 self-antigens, respectively, that are linked to a variety of autoimmune diseases (Fig. 1). See

167 Supplementary Table 1_for autoantibody levels, Supplementary Table 2 for their

168 abbreviations and targets, and Supplementary Table 3 for autoantibody and disease association.

169 Multi-study factor analysis (MSFA) (Fig. 2 and Supplementary Table 6) revealed a

170 progressive dysregulation in the number of latent factors from mild COVID-19 patients to those

171 with moderate and severe disease. The two latter groups presented fewer latent factors when

172 compared with control and mild COVID-19 groups, indicating by this statistical inference

173 approach that the perturbation of normal autoantibody levels mostly occurs in patients with

174 moderated and severe COVID-19.

175 We found significantly elevated levels of IgG (Supplementary Fig. 1) and IgA

176 (Supplementary Fig. 2) autoantibodies targeting a total of 42 and 25 antigens, respectively,

177 when comparing COVID-19 groups (mild, moderate, and severe) versus healthy controls (Fig.

178 3a). In general, while patients with mild COVID-19 exhibited only slight differences in the

179 serum levels of autoantibodies when compared to healthy controls, patients with moderate

180 COVID-19 exhibited more evident differences, with the highest levels of autoantibodies noted

181 in the severe group. In agreement with the results obtained by MSFA, we observed a progressive 
182 increase of the autoantibodies targeting autoantigens such as those associated with Alzheimer's

183 disease (rabaptin-5, tau protein), antiphospholipid syndrome (cardiolipin), multiple sclerosis and

184 other neuroimmune disorders (myelin basic protein), celiac disease (transglutaminases, zonulin),

185 neuropsychiatric and neurodegenerative disorders (NMDAR), gut motility disorders (enteric

186 nerve), liver autoimmunity (liver microsomal antigen), immune thrombocytopenia (platelet

187 glycoprotein), myasthenia gravis (acetylcholine receptor, somatotropin), neurodegenerative

188 diseases (neurofilament proteins), Parkinson's disease ( $\alpha$-synuclein), pemphigus vulgaris or

189 foliaceus (Desmoglein-E-Cadherin 1), neurological disorders (S100B), rheumatic heart disease

190 ( $\alpha$-myosin), rheumatoid arthritis ( $\alpha$-enolase, fibulin), systemic lupus erythematosus (cardiolipin,

191 dsDNA, rabaptin-5), type 1 diabetes (islet cell antigen), immune thrombotic thrombocytopenia

192 (heparin), and vitiligo (tyrosinase) (Supplementary Table 3, Supplementary Fig. 1-2 and Fig.

193 3a, 3d-e). In addition, we also identified reduced levels of IgG autoantibodies such as those

194 against a-myosin, rabaptin-5, S100B, and cerebellar as well as IgA autoantibodies targeting the

195 NMDA R and cerebellar. Thus, indicating a broad breakdown of physiological autoantibody

196 levels/self-tolerance in patients with COVID-19 that paralleled disease severity. Of note, the

197 progressive perturbation of autoantibody levels was accompanied by increased serum

198 concentration of anti-SARS-CoV-2 antibodies (Fig. 3e).

Fig. 3b and Fig. 3c display the functional associations of the autoantibody targets, which

200 are represented by functional enrichment analysis (gene ontology or GO). These results indicate

201 that, among others, COVID-19 patients have dysregulated levels of autoantibodies affecting in

202 neurological functions such as memory, learning, and cognition ${ }^{66-68}$ (Supplementary Table 4

203 and Supplementary Fig. 3). Taken together, our data indicate that autoantibodies may also be 
204 involved with altered learning, memory and neuroplasticity ${ }^{68}$ recently reported during SARS-

205 CoV-2 infection, which promotes a systemic inflammation milieu that might be neurotoxic.

Autoantibody generation correlates with severe SARS-CoV-2 infection and anosmia

Based on the concept that signatures of autoantibody correlations are associated with

209 physiological and pathological immune homeostasis ${ }^{10,12,40}$, we investigated the correlation

210 between signatures of the $\operatorname{IgA}$ and $\operatorname{IgG}$ autoantibodies and disease severity. Bivariate correlation

211 analysis revealed (Supplementary Table 5 and Fig. 4a) that patients with mild COVID-19

212 exhibited few differences in the autoantibody correlation signatures when compared to healthy

213 controls. In contrast, patients with moderate COVID-19 started to exhibit new positive and

214 negative correlations between autoantibodies while the severe group displayed the most disparate

215 topological correlation pattern, increasingly with preferentially positive correlations between

216 autoantibodies.

217 We then assessed whether anosmia (loss of smell), which was one of the most consistent

218 symptoms associated with SARS-CoV-2 infections ${ }^{69}$, also correlated with autoantibody

219 signatures in patients with mild or moderate COVID-19. Due to the extreme clinical condition

220 requiring oxygen replacement therapy that precludes the characterization of anosmia, patients

221 with severe COVID-19 were excluded from this analysis. We observed that the interconnections

222 among the autoantibodies are reduced in both mild and moderate COVID-19 with anosmia when

223 compared with those without (Supplementary Fig. 4e and Fig. 3b). Taken together, this

224 suggests the presence of multilayered factors that influence the autoantibody signatures,

225 including both disease severity and anosmia that affect relationships among autoantibodies

226 differently. 


\section{Ranking autoantibodies as predictors of COVID-19 severity}

229 To investigate the classification power of autoantibodies, we used random forest modeling,

230 which predicts outcomes based on decision trees ${ }^{70}$, to rank the top 10 autoantibodypredictors of

231 COVID-19 outcomes. Random forest classification of COVID-19 patients presented a stable

232 curve showing the highest error rate (out-of-bag OOB) for the severe COVID-19 group based on

233 both IgA or IgG autoantibodies (Supplementary Fig. 6a, 6d, 6g, and 6h). The confusion matrix

234 showed that the random forest model was unable to distinguish moderate from severe COVID-19

235 groups (Supplementary Fig. 6b and 6e). These results indicate that moderate and severe

236 COVID-19 patients may present a similar autoantibody signature. Therefore, we lumped

237 moderate and severe COVID-19 patients into the same group. Using this approach, the out-of-

238 bag (OOB) estimate of the error rate for all groups changed from $37 \%$ to $22 \%$ for $\operatorname{IgA}($ Fig. 5d)

239 and from $25 \%$ to $12 \%$ for IgG autoantibodies (Fig. 5a). This way, the model was considered

240 adequate to classify the COVID-19 patients by severity according to IgA and IgG autoantibodies,

241 showing areas under the ROC curve of $0.91 \%, 0.85 \%$, and $0.90 \%$ for the healthy control,

242 moderate, and severe COVID-19 groups for IgA (Supplementary Fig. 6j) and 0.98\%. 0.96\%

243 and $0.99 \%$ for IgG (Supplementary Fig. 6i). In this context, autoantibodies tracked with anti-

244 SARS-CoV-2 antibodies and age as predictive factors for COVID-19 outcome. Among them are

245 IgG autoantibodies targeting the NMDA R, insulin, PG, LSPC, human RO60, claudin, heparin,

246 and S100B, as well as IgA autoantibodies targeting cardiolipin, the NMDA R, HEK, cerebellar,

247 ICA, AbP, DEC, $\alpha$-synucleins, and tyrosinase. Thus, IgA and IgG autoantibodies stratify

248 COVID-19 according to disease severity. 
250 outcomes, we performed principal component analysis (PCA) with a spectral decomposition

251 approach $^{40,41}$ to analyze whether the top 10 ranked variables (IgA and $\operatorname{IgG}$ autoantibodies and 252 age) as predictors of COVID-19 severity have stratification power. This approach indicated, in

253 accordance with the random forest modeling, that the ranked autoantibodies stratify COVID-19

254 patients according to disease severity. (Fig. 5g and 5h). While healthy controls and patients with 255 mild COVID-19 presented with similar autoantibody patterns, moderate and severe COVID-19 256 groups clustered closely. Together, these results indicate that the immune responses against 257 SARS-CoV-2 not only are associated with an increase of serum autoantibody levels linked to 258 autoimmune diseases, but also have predictive stratification values for COVID-19 patients.

SARS-CoV-2 infection dysregulates autoantibody levels in an age-dependent manner

Considering age as a major predictor of severe outcomes in COVID-19, which was also

262 identified by our data (Random forest) ${ }^{71}$, we investigated the effect of age on $\mathrm{f}$ the top $\operatorname{IgG}$ and

263 IgA autoantibody predictors of COVID-19 severity. We classified control and disease groups by 264 age (young $<60$ and elderly $\geq 60$ ) and found that both moderately and severely affected elderly 265 COVID-19 patients show an overall tendency to have elevated autoantibody levels when 266 compared with younger patients (Fig. 6a). However, only elderly patients with severe COVID26719 presented with significant differences in most of the top $10 \operatorname{IgG}$ (Fig. 6b) and $\operatorname{IgA}($ Fig. 6c) 268 autoantibodies when compared with young patients with severe disease. Of note, except for 269 autoantibodies targeting $\alpha$-enolase, cardiolipin, CS, DEC, dsDNA, ENA, elderly patients with 270 severe COVID-19 had significant differences in all other IgG autoantibodies (Supplementary 271 Fig. 7a). In turn, IgA autoantibodies against 10 antigens displayed significant differences 
273 No age-dependent differences were observed regarding the levels of anti-SARS-CoV-2 274 antibodies.

275 Of note, there were only a few (IgG autoantibodies targeting heparin and LSPC; SARS-

276 CoV-2 antibodies; IgA autoantibodies targeting cardiolipin, DEC, ICA, and tyrosinase) age-

277 dependent significant differences in patients with anosmia versus no anosmia regarding the top

27810 autoantibody predictors of COVID-19 severity (Supplementary Fig. 8). Furthermore, we 279 asked whether sex also impacts the levels of autoantibodies during SARS-Co-2 infections. IgG 280 anti-cardiolipin-aab was elevated in males from all three disease groups (but only significantly in 281 mild and moderate COVID-19 cohorts) when compared to females. Except for this, there were 282 no significant sex differences in the levels of the top $10 \mathrm{IgG}$ and $\operatorname{IgA}$ autoantibody predictors of 283 COVID-19 severity (Supplementary Fig. 9).

\section{DISCUSSION}

This work sheds light on the immunopathogenesis of COVID-19 demonstrating the

287 presence of increased levels of $\operatorname{IgG}$ and $\operatorname{IgA}$ autoantibodies linked to diverse number of 288 autoimmune diseases. Among them, are autoantibodies not yet reported in COVID-19 patients 289 such as those targeting neuronal antigens (e.g., $\alpha$-synuclein, acetylcholine receptor, myeloid- $\beta$ 290 peptide, $\beta$-catenin, brain-derived neurotrophic factor, cerebellar antigen, chondroitin sulphate, 291 dopamine receptors [D1 and D2], enteric nerve, ganglioside, glutamic acid decarboxylase, 292 myelin basic protein, myelin oligodendrocyte glycoprotein, neurofilament proteins, NMDA 293 receptor, rabaptin-5, somatotropin, S100B, tau protein, and transglutaminases 6) and non294 neuronal antigens (e.g., $\alpha$-enolase, $\alpha$-myosin, claudin-5 and -7, collagen, desmoglein-E-cadherin 

islet cell antigen, occluding, platelet glycoprotein, transglutaminases 2 and 3, tyrosinase, and 297 zonulin).

such as disease severity and anosmia. Besides expanding the spectrum of autoantibodies 300 associated with COVID-19 (e.g., cardiolipin $^{72}$, dsDNA ${ }^{72}$, epithelial cell antigen ${ }^{73}$, heparin ${ }^{74}$, 301 human $\mathrm{RO}^{75}$, liver microsomal antigen ${ }^{40}$, lung surfactant protein $\left.\mathrm{C}\right)^{76}$, our data also indicate 302 that both disease severity and anosmia disrupt the physiological $\operatorname{IgG}$ and $\operatorname{IgA}$ autoantibody 303 signatures. These findings are consistent with the notion that autoantibodies are natural 304 components of human physiology and become dysregulated under pathological 305 conditions ${ }^{19,20,77,78}$. Further supporting the concept that COVID-19 disrupts antibody physiology, 306 anti-SARS-CoV-2 antibody levels accompany the levels of autoantibodies. Those antibodies 307 targeting self- and non-self-antigens, along with age, are predictors of disease severity as 308 classified by random forest modeling. Viral-induced autoimmune diseases are due to multiple 309 mechanisms such as molecular mimicry ${ }^{79}$, epitope spreading ${ }^{80}$, and bystander activation, all of 310 which could possibly be involved in SARS-CoV-2 induced autoimmunity. Indeed, evidence has 311 been provided for strong, chronic inflammation promoting the release of self-antigens and high 312 cytokine levels activating bystander T-cells ${ }^{81}$, and for molecular mimicry ${ }^{82-84}$, all of which have

313 been associated with severe COVID-19. Molecular mimicry has been explored by assessing the 314 SARS-CoV-2 proteome, which revealed the existence of 21 viral peptides that show at least $90 \%$

315 homology with human proteins known to be involved in autoimmune diseases such as multiple 316 sclerosis and rheumatoid arthritis ${ }^{82}$. Furthermore, it has been shown that monoclonal antibodies 317 generated against SARS-CoV-2 proteins reacted with 28 out of 55 tested autoantigens ${ }^{70-71}$. These 
observations may explain why the multiple serum autoantibodies causative to autoimmune

319 diseases dysregulate their levels, perturbing their physiologic function. These findings are in line

320 with reports from ours ${ }^{20}$ and other groups ${ }^{10,40}$ demonstrating that changes in autoantibody

321 signatures affect physiologic and pathologicl immune homeostasis ${ }^{20}$.

Of note, the random forest model suggests that anti-SARS-CoV-2 antibodies and autoantibodies, together with the major risk factor age ${ }^{71}$, are important predictors of COVID-19 severity. Our finding that the highest levels of anti-SARS-CoV-2 antibodies are achieved by patients with severe COVID-19 is in agreement with previous reports demonstrating a similar 326 pattern of humoral immune response in critically ill COVID-19 patients ${ }^{75,85}$. In addition, it was

327 shown that the levels of anti-SARS-CoV-2 antibodies accompany longitudinally with the 328 dysregulation of autoantibody levels ${ }^{40}$. While we found no age effect on the levels of anti-SARS-

329 CoV-2 antibodies, severe SARS-CoV-2 infection induces higher autoantibody levels in elderly 330 patients compared with young patients. Thus, our data point to novel mechanisms involved in the 331 risk intersection of immunosenescence and COVID-19 ${ }^{71}$, suggesting that SARS-CoV-2

332 infection induces a broader loss of self-tolerance than previously thought, particularly in elderly

333 patients. In this context, several age-associated factors such as chronic inflammation in ageing

334 (inflammageing ${ }^{86}$ ) might promote the production of autoantibodiesas well as the tendency to 335 naturally progress to immune dysregulation of innate ${ }^{87}$ and adaptive ${ }^{88}$ immune cells. Therefore, 336 our data support the idea that the induction of high levels of serum autoantibodies in elderly 337 COVID-19 patients contribute to the increased risk for negative outcomes in older persons ${ }^{89}$.

338 Because autoantibodies are strong predictors of COVID-19 severity, our findings expand

339 the number of autoantibodies that qualify as biomarkers for the severity of SARS-CoV-2 340 infection. Among them are those targeting molecules involved in neurological functions (e.g., 
341 cognition, learning, memory, synaptic transmission) and glucose metabolism. For instance, while

342 anti-cardiolipin antibodies were previously associated with the development of

343 hyperinflammatory syndromes ${ }^{90}$, the high levels of autoantibodies targeting neuronal-associated

344 molecules could provide clues why the respiratory symptoms of COVID-19 patients are often

345 accompanied by short- and long-term neuropsychiatric symptoms and brain sequelae ${ }^{68}$. It has

346 been convincingly shown that SARS-CoV-2 is able enter the brain by crossing the blood-brain

347 barrier $(\mathrm{BBB})$ because inflammatory cytokines induce BBB instability ${ }^{68,91,92}$. Our data indicate

348 that the array of autoantibodies targeting neuronal molecules is an additional molecular layer that

349 could contribute to the neurological manifestations ${ }^{66,67}$ occuring in COVID-19 patients.

There are limitations of our worlk that need consideration. For instance, our study did not

351 have longitudinal data to analyze the pharmacokinetics of the IgG and IgA autoantibodies, from

352 disease onset to convalescence or post-acute COVID-19 syndrome. Our study cohort did not

353 include asymptomatic patients. Moreover, we cannot exclude the possibility that at least some of

354 our patients had high levels of autoantibodies prior to SARS-CoV-2 infection, or that

355 autoantibodies to some antigens (e.g., heparin) could have been induced by anticoagulant therapy

356 with heparin. In addition, we did not assess alterations in the number of circulating B

357 lymphocytes and whether this could explain the higher serum levels of autoantibodies. In

358 addition, future studies are required to clarify the role of virus and host genetics in the production

359 of autoantibodies. On the other hand, our work raises new questions such as whether the

360 dysregulated levels of autoantibodies remain after COVID-19 remission and these levels are in

361 patients with post-COVID-19 syndrome.

362 Taken together, our work provides a comprehensive view of the spectrum of

363 autoantibodies linked with autoimmune diseases that are induced by SARS-CoV-2 infection. 
364 This work maps the intersection of COVID-19 and autoimmunity ${ }^{93-95}$, demonstrating the 365 dysregulation of multiple autoantibodies that are linked to autoimmune diseases during SARS-

366 CoV-2 infections, and the altered correlation signatures according to disease severity and

367 anosmia. The data presented suggest a gradual involvement of autoantibodies in the severity of

368 COVID-19. We identified several new clinically relevant autoantibodies that can be used as

369 biomarkers that predict COVID-19 severity and to open avenues to develop new treatment

370 strategies. 


\section{Acknowledgments}

373 We acknowledge the patients for participation in this study. We would like to acknowledge the 374 contributions of Lev Rochel Bikur Cholim of Lakewood (led by Rabbi Yehuda Kasirer and Mrs. 375 Leeba Prager) and their hundreds of volunteers who participated in collecting samples for this 376 research through the MITZVA Cohort. We thank Immunosciences and Cyrex Laboratories for 377 financial support and INOVA Diagnostics for providing their diagnostic ELISA kits for 378 autoimmunity at a very discounted rate Furthermore, we would like to thank Vilma Samayoa, 379 David Cisneros, Roberto Melgar, Dana Ashley Hill and Amanda Thornton for technical assistance.. We also thank the São Paulo Research Foundation (FAPESP grants: 2020/07972-1 to GCB; 2020/09146-1 to PPF; 2020/11710-2 to DRP, 2018/18886-9, 2020/01688-0, and 2020/07069-0 to OCM), and the Coordination for the Improvement of Higher Education Personnel (CAPES) Financial Code 001 (grant to ISF) for financial support.

\section{Data availability}

386 All data present in this manuscript are provided as Supplementary files. All R packages used in 387 this manuscript are are available at the following link: 388 https:/github.com/gabrielacbaiocchi/Autoantibodies-linked-to-autoimmune-diseases-associate389 with-COVID-19-outcomes.git

\section{Author contributions}

392 YS, AZR, IZ, AV, and EV conceived the project; YS, AZR, IZ, and GH designed the study. JIS, 393 AZR, IZ, and MTL diagnosed, recruited or followed-up the patients. AV and EV performed the 394 experiments. AZR, DAH, AT, GH, JZ, JIS, AZR, IZ, MTL, EV, YBL, HA, and YS coordinated 395 the serum collection and databank. GCB, AHCM, ISF, DLMF, DRP, PPF, MK, RDV, and OCM 396 performed data and bioinformatics analyses. GCB, AV, LMG, HDO, and OCM wrote the 397 manuscript. JS, HH, YO, AZR, VLGC, NOSC, RDV, IZ, GCB, AV, LFS, LMG, HDO, and 398 OCM edited the manuscript; GCB, AV, AZR, YO, LFS, IZ, AHCM, EV, DAH, AT, LMG, 399 YBL, JIS, JZ, NOSC, VLGC, HH, GR, HA, OCM, and YS, provided scientific insights. 
medRxiv preprint doi: https://doi.org/10.1101/2022.02.17.22271057; this version posted February 18, 2022. The copyright holder for this preprint (which was not certified by peer review) is the author/funder, who has granted medRxiv a license to display the preprint in perpetuity.

\section{It is made available under a CC-BY-NC-ND 4.0 International license .}

\section{Competing interests}

402 The authors declare no competing interests.

403 


\section{REFERENCES}

1. WHO Coronavirus (COVID-19) Dashboard | WHO Coronavirus (COVID-19) Dashboard With Vaccination Data.

2. Gorbalenya, A. E. et al. The species Severe acute respiratory syndrome-related coronavirus: classifying 2019-nCoV and naming it SARS-CoV-2. Nat. Microbiol. 202054 5, 536-544 (2020).

3. Konings, F. et al. SARS-CoV-2 Variants of Interest and Concern naming scheme conducive for global discourse. Nat. Microbiol. 6, 821-823 (2021).

4. Maslo, C. et al. Characteristics and Outcomes of Hospitalized Patients in South Africa During the COVID-19 Omicron Wave Compared With Previous Waves. JAMA (2021) doi:10.1001/JAMA.2021.24868.

5. Hacisuleyman, E. et al. Vaccine Breakthrough Infections with SARS-CoV-2 Variants. $N$. Engl. J. Med. 384, 2212-2218 (2021).

6. Karim, S. S. A. \& Karim, Q. A. Omicron SARS-CoV-2 variant: a new chapter in the COVID-19 pandemic. Lancet 398, 2126-2128 (2021).

7. Webb, B. J. et al. Clinical criteria for COVID-19-associated hyperinflammatory syndrome: a cohort study. Lancet. Rheumatol. 2, e754-e763 (2020).

8. Koutsakos, M. et al. Integrated immune dynamics define correlates of COVID-19 severity and antibody responses. Cell reports. Med. 2, (2021).

9. Lourda, M. et al. High-dimensional profiling reveals phenotypic heterogeneity and disease-specific alterations of granulocytes in COVID-19. Proc. Natl. Acad. Sci. U. S. A. 118, (2021).

10. Wang, E. Y. et al. Diverse functional autoantibodies in patients with COVID-19. Nat. 20215957866 595, 283-288 (2021).

11. Bastard, P. et al. Autoantibodies against type I IFNs in patients with life-threatening COVID-19. Science (80-. ). 370, (2020).

12. Cabral-Marques, O. et al. The relationship between autoantibodies targeting GPCRs and the renin-angiotensin system associates with COVID-19 severity. medRxiv 2021.08.24.21262385 (2021) doi:10.1101/2021.08.24.21262385.

13. Khamsi, R. Rogue antibodies could be driving severe COVID-19. Nature 590, 29-31 (2021).

14. Ehrenfeld, M. et al. Covid-19 and autoimmunity. Autoimmun. Rev. 19, (2020).

15. Lichtman, A. H. Anti-body antibodies in COVID-19. Sci. Immunol. 6, (2021).

16. Dotan, A. et al. The SARS-CoV-2 as an instrumental trigger of autoimmunity. Autoimmun. Rev. 20, (2021).

17. Halpert, G. \& Shoenfeld, Y. SARS-CoV-2, the autoimmune virus. Autoimmun. Rev. 19, 102695 (2020).

18. Amendt, T. et al. Autoreactive antibodies control blood glucose by regulating insulin homeostasis. Proc. Natl. Acad. Sci. 119, e2115695119 (2022).

19. Nagele, E. P. et al. Natural IgG Autoantibodies Are Abundant and Ubiquitous in Human Sera, and Their Number Is Influenced By Age, Gender, and Disease. PLoS One 8, e60726 (2013).

20. Cabral-Marques, O. et al. GPCR-specific autoantibody signatures are associated with physiological and pathological immune homeostasis. Nat. Commun. 9, 5224 (2018).

21. Lutz, H. U., Binder, C. J. \& Kaveri, S. Naturally occurring auto-antibodies in homeostasis 
and disease. Trends Immunol. 30, 43-51 (2009).

22. Leslie, D., Lipsky, P. \& Louis Notkins, A. Autoantibodies as predictors of disease. J. Clin. Invest. 108, 1417 (2001).

23. Deane, K. D. \& El-Gabalawy, H. Pathogenesis and prevention of rheumatic disease: focus on preclinical RA and SLE. Nat. Rev. Rheumatol. 2014104 10, 212-228 (2014).

24. Pedroza-Pacheco, I. \& Borrow, P. Targeting autoantibodies in COVID-19. Nat. Rev. Immunol. 2021213 21, 134-134 (2021).

25. Pavlovic, M., Kats, A., Cavallo, M. \& Shoenfeld, Y. Clinical and Molecular Evidence for Association of SLE with parvovirus B19. Lupus 19, 783-792 (2010).

26. Maya, R., Gershwin, M. E. \& Shoenfeld, Y. Hepatitis B virus (HBV) and autoimmune disease. Clin. Rev. Allergy Immunol. 34, 85-102 (2008).

27. Barzilai, O. et al. Epstein-Barr virus and cytomegalovirus in autoimmune diseases: are they truly notorious? A preliminary report. Ann. N. Y. Acad. Sci. 1108, 567-577 (2007).

28. Barzilai, O., Ram, M. \& Shoenfeld, Y. Viral infection can induce the production of autoantibodies. Curr. Opin. Rheumatol. 19, 636-643 (2007).

29. Lucchese, G. Cerebrospinal fluid findings in COVID-19 indicate autoimmunity. The Lancet. Microbe 1, e242 (2020).

30. Taha, M. \& Samavati, L. Antiphospholipid antibodies in COVID-19: a meta-analysis and systematic review. RMD Open 7, e001580 (2021).

31. Khan, F. et al. COVID-19-associated Guillain-Barre syndrome: Postinfectious alone or neuroinvasive too? J. Med. Virol. 93, 6045-6049 (2021).

32. Gutiérrez-Ortiz, C. et al. Miller Fisher syndrome and polyneuritis cranialis in COVID-19. Neurology 95, e601-e605 (2020).

33. Boddu, S. K., Aurangabadkar, G. \& Kuchay, M. S. New onset diabetes, type 1 diabetes and COVID-19. Diabetes Metab. Syndr. 14, 2211-2217 (2020).

34. Jacobs, J. \& Eichbaum, Q. COVID-19 associated with severe autoimmune hemolytic anemia. Transfusion 61, 635-640 (2021).

35. Mateu-Salat, M., Urgell, E. \& Chico, A. SARS-COV-2 as a trigger for autoimmune disease: report of two cases of Graves' disease after COVID-19. J. Endocrinol. Invest. 43, 1527-1528 (2020).

36. Bhattacharjee, S. \& Banerjee, M. Immune Thrombocytopenia Secondary to COVID-19: a Systematic Review. Sn Compr. Clin. Med. 2, 1 (2020).

37. Parisi, S., Borrelli, R., Bianchi, S. \& Fusaro, E. Viral arthritis and COVID-19. Lancet Rheumatol. 2, e655-e657 (2020).

38. Zamani, B., Moeini Taba, S. M. \& Shayestehpour, M. Systemic lupus erythematosus manifestation following COVID-19: a case report. J. Med. Case Rep. 15, 1-4 (2021).

39. Woodruff, M. C. et al. Relaxed peripheral tolerance drives broad de novo autoreactivity in severe COVID-19. medRxiv Prepr. Serv. Heal. Sci. (2021) doi:10.1101/2020.10.21.20216192.

40. Chang, S. E. et al. New-onset IgG autoantibodies in hospitalized patients with COVID-19. Nat. Commun. 2021121 12, 1-15 (2021).

41. Zuo, Y. et al. Prothrombotic autoantibodies in serum from patients hospitalized with COVID-19. Sci. Transl. Med. 12, 3876 (2020). 
43. Losartan for Patients With COVID-19 Requiring Hospitalization - Full Text View ClinicalTrials.gov.

44. Cavalli, E. et al. Entangling COVID-19 associated thrombosis into a secondary antiphospholipid antibody syndrome: Diagnostic and therapeutic perspectives (Review). Int. J. Mol. Med. 46, 903-912 (2020).

45. Galeotti, C. \& Bayry, J. Autoimmune and inflammatory diseases following COVID-19. Nat. Rev. Rheumatol. 2020168 16, 413-414 (2020).

46. Ramos-Casals, M., Brito-Zerón, P. \& Mariette, X. Systemic and organ-specific immunerelated manifestations of COVID-19. Nat. Rev. Rheumatol. 2021 176 17, 315-332 (2021).

47. Silverberg, J. et al. Association of Varying Clinical Manifestations and Positive AntiSARS-CoV-2 IgG Antibodies: A Cross-Sectional Observational Study. J. allergy Clin. Immunol. Pract. (2021) doi:10.1016/J.JAIP.2021.06.046.

48. Zyskind, I. et al. SARS-CoV-2 Seroprevalence and Symptom Onset in Culturally Linked Orthodox Jewish Communities Across Multiple Regions in the United States. JAMA Netw. open 4, (2021).

49. COVID-19 Clinical management: living guidance.

50. Baggi, F. et al. Breakdown of Tolerance to a Self-Peptide of Acetylcholine Receptor $\alpha-$ Subunit Induces Experimental Myasthenia Gravis in Rats. J. Immunol. 172, 2697-2703 (2004).

51. Vojdani, A. \& Turnpaugh, C. C. Antibodies against Group A Streptococcus, dopamine receptors, and ganglioside GM1 cross-react with a variety of food antigens, potentially interfering with biomarkers for PANS and PANDAS. Biomarkers in Neuropsychiatry 3 , 100023 (2020).

52. Swedo, E. et al. Brain Human Monoclonal Autoantibody from Sydenham Chorea Targets Dopaminergic Neurons in Transgenic Mice and Signals Dopamine D2 Receptor:

Implications in Human Disease. J. Immunol. 191, 5524-5541 (2013).

53. Vojdani, A. \& Vojdani, E. Amyloid-Beta 1-42 Cross-Reactive Antibody Prevalent in Human Sera May Contribute to Intraneuronal Deposition of A-Beta-P-42. Int. J. Alzheimers. Dis. 2018, (2018).

54. Wood, J. D., Liu, S., Drossman, D. A., Ringel, Y. \& Whitehead, W. E. Anti-Enteric Neuronal Antibodies and the Irritable Bowel Syndrome. J Neurogastroenterol Motil 18, 78-85 (2012).

55. Demchyshyn, L. L., Sugamori, K. S., Lee, F. J. S., Hamadanizadeh, S. A. \& Niznik, H. B. The Dopamine D1D Receptor: CLONING AND CHARACTERIZATION OF THREE PHARMACOLOGICALLY DISTINCT D1-LIKE RECEPTORS FROM Gallus domesticus(*). J. Biol. Chem. 270, 4005-4012 (1995).

56. Omdal, R. et al. Neuropsychiatric disturbances in SLE are associated with antibodies against NMDA receptors. Eur. J. Neurol. 12, 392-398 (2005).

57. Kaniakova, M. et al. Key amino acid residues within the third membrane domains of NR1 and NR2 subunits contribute to the regulation of the surface delivery of N-methyl-Daspartate receptors. J. Biol. Chem. 287, 26423-26434 (2012).

58. Vojdani, A., Vojdani, E., Saidara, E. \& Kharrazian, D. Reaction of Amyloid- $\beta$ Peptide Antibody with Different Infectious Agents Involved in Alzheimer's Disease. $J$. Alzheimers. Dis. 63, 847-860 (2018).

541 60. Vito, R. De, Bellio, R., Trippa, L. \& Parmigiani, G. Bayesian multistudy factor analysis 
for high-throughput biological data. https://doi.org/10.1214/21-AOAS1456 15, 1723-1741 (2021).

61. De Vito, R., Bellio, R., Trippa, L. \& Parmigiani, G. Multi-study Factor Analysis. (2016).

62. Lever, J., Krzywinski, M. \& Altman, N. Points of Significance: Principal component analysis. Nature Methods vol. 14 641-642 (2017).

63. Ringnér, M. What is principal component analysis? Nature Biotechnology vol. 26 303304 (2008).

64. Kotlyar, M. et al. IID 2021: towards context-specific protein interaction analyses by 13-14 (2021).

65. Liaw, A. \& Wiener, M. (8) (PDF) Classification and Regression by RandomForest.

66. Becker, J. H. et al. Assessment of Cognitive Function in Patients After COVID-19 Infection. JAMA Netw. Open 4, e2130645-e2130645 (2021).

67. Alnefeesi, Y. et al. Impact of SARS-CoV-2 Infection on Cognitive Function: A Systematic Review. Front. Psychiatry 11, 1629 (2021).

68. Boldrini, M., Canoll, P. D. \& Klein, R. S. How COVID-19 Affects the Brain. JAMA Psychiatry 78, 682-683 (2021).

69. Sudre, C. H. et al. Anosmia, ageusia, and other COVID-19-like symptoms in association with a positive SARS-CoV-2 test, across six national digital surveillance platforms: an observational study. Lancet. Digit. Heal. 3, e577-e586 (2021).

70. Liaw, A. \& Wiener, M. Classification and Regression by randomForest. 2, (2002).

71. Bartleson, J. M. et al. SARS-CoV-2, COVID-19 and the aging immune system. Nat. Aging 202119 1, 769-782 (2021).

72. Lerma, L. A. et al. Prevalence of autoantibody responses in acute coronavirus disease 2019 (COVID-19). J. Transl. Autoimmun. 3, (2020).

73. Yang, Y. H. et al. Autoantibodies against human epithelial cells and endothelial cells after severe acute respiratory syndrome (SARS)-associated coronavirus infection. J. Med. Virol. 77, 1-7 (2005).

74. Pang, E., Ghosh, S., Chemmanam, T., Grove, C. \& Phillips, T. Cerebral arterial and venous thrombosis due to COVID-19 vaccine-induced immune thrombotic thrombocytopenia. BMJ Case Rep. 15, e245445 (2022).

75. Lerma, L. A. et al. Prevalence of autoantibody responses in acute coronavirus disease 2019 (COVID-19). J. Transl. Autoimmun. 3, 100073 (2020).

76. Sinnberg, T. et al. IgA autoantibodies target pulmonary surfactant in patients with severe COVID-19. medRxiv 2021.02.02.21250940 (2021) doi:10.1101/2021.02.02.21250940.

77. Cabral-Marques, O. \& Riemekasten, G. Functional autoantibodies targeting G proteincoupled receptors in rheumatic diseases. Nat. Rev. Rheumatol. 20171311 13, 648-656 (2017).

78. Watanabe, M. et al. Anti-cytokine autoantibodies are ubiquitous in healthy individuals. FEBS Lett. 581, 2017-2021 (2007).

79. Rojas, M. et al. Molecular mimicry and autoimmunity. J. Autoimmun. 95, 100-123 (2018).

80. Powell, A. M. \& Black, M. M. Epitope spreading: protection from pathogens, but propagation of autoimmunity? Clin. Exp. Dermatol. 26, 427-433 (2001).

81. Gregorova, M. et al. Post-acute COVID-19 associated with evidence of bystander T-cell activation and a recurring antibiotic-resistant bacterial pneumonia. Elife 9, 1-13 (2020). 
82. Karami Fath, M. et al. SARS-CoV-2 Proteome Harbors Peptides Which Are Able to Trigger Autoimmunity Responses: Implications for Infection, Vaccination, and Population Coverage. Front. Immunol. 12, 3174 (2021).

83. Vojdani, A., Vojdani, E. \& Kharrazian, D. Reaction of Human Monoclonal Antibodies to SARS-CoV-2 Proteins With Tissue Antigens: Implications for Autoimmune Diseases. Front. Immunol. 11, (2021).

84. Vojdani, A. \& Kharrazian, D. Potential antigenic cross-reactivity between SARS-CoV-2 and human tissue with a possible link to an increase in autoimmune diseases. Clin. Immunol. 217, (2020).

85. Yan, X. et al. Anti-SARS-CoV-2 IgG levels in relation to disease severity of COVID-19. J. Med. Virol. 94, 380-383 (2022).

86. Ferrucci, L. \& Fabbri, E. Inflammageing: chronic inflammation in ageing, cardiovascular disease, and frailty. Nat. Rev. Cardiol. 2018159 15, 505-522 (2018).

87. Shaw, A. C., Goldstein, D. R. \& Montgomery, R. R. Age-dependent dysregulation of innate immunity. Nat. Rev. Immunol. 20131312 13, 875-887 (2013).

88. Rocamora-Reverte, L., Melzer, F. L., Würzner, R. \& Weinberger, B. The Complex Role of Regulatory T Cells in Immunity and Aging. Front. Immunol. 11, 3566 (2021).

89. Poletti, P. et al. Association of Age With Likelihood of Developing Symptoms and Critical Disease Among Close Contacts Exposed to Patients With Confirmed SARS-CoV2 Infection in Italy. JAMA Netw. Open 4, e211085-e211085 (2021).

90. Xu, Y. M., Chu, Y. Q. \& Wang, H. Correlation Analysis of Anti-Cardiolipin Antibody/D Dimer/C-Reactive Protein and Coronary Artery Lesions/Multiple-Organ Damage in Children With Kawasaki Disease. Front. Pediatr. 9, 1001 (2021).

91. Zhang, L. et al. SARS-CoV-2 crosses the blood-brain barrier accompanied with basement membrane disruption without tight junctions alteration. Signal Transduct. Target. Ther. $2021616,1-12$ (2021).

92. Rhea, E. M. et al. The S1 protein of SARS-CoV-2 crosses the blood-brain barrier in mice. Nat. Neurosci. 2020243 24, 368-378 (2020).

93. Liu, Y., Sawalha, A. H. \& Lu, Q. COVID-19 and autoimmune diseases. Curr. Opin. Rheumatol. 33, 155-162 (2021).

94. Knight, J. S. et al. The intersection of COVID-19 and autoimmunity. J. Clin. Invest. 131, (2021).

95. Saad, M. A. et al. COVID-19 and Autoimmune Diseases: A Systematic Review of Reported Cases. Curr. Rheumatol. Rev. 17, 193-204 (2021). 


\section{Data acquisition}

\begin{tabular}{ccccc}
\multicolumn{2}{c}{ Study cohort } & Sample collection & $\begin{array}{c}\text { Autoantibody detection } \\
\text { by ELISA }\end{array}$ \\
Healthy & \multicolumn{2}{c}{ COVID-19 } &
\end{tabular}

\section{Data analysis}

Multi-Study Factor Analysis (MSFA)

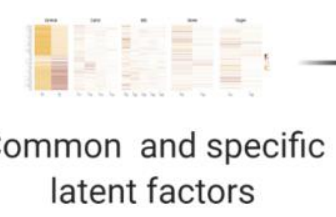

Wilcoxon rank sum test

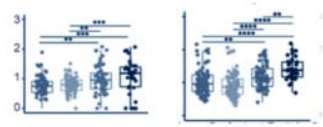

Differences in aab levels between groups
GO analysis

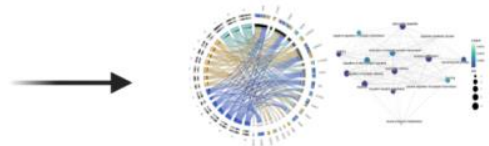

Functional enrichment of aab targets

\section{Data analysis}

Bivariate and multivariate correlation analysis

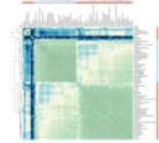

Aab correlation signatures according to patient groups

\section{Random Forest}

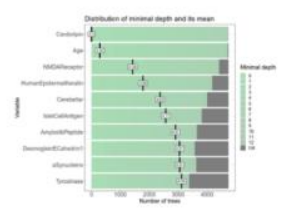

Ranking aab as predictors of COVID-19 severity
Principal Component Analysis

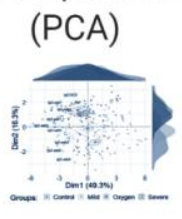

Stratification of patients according to disease severity 


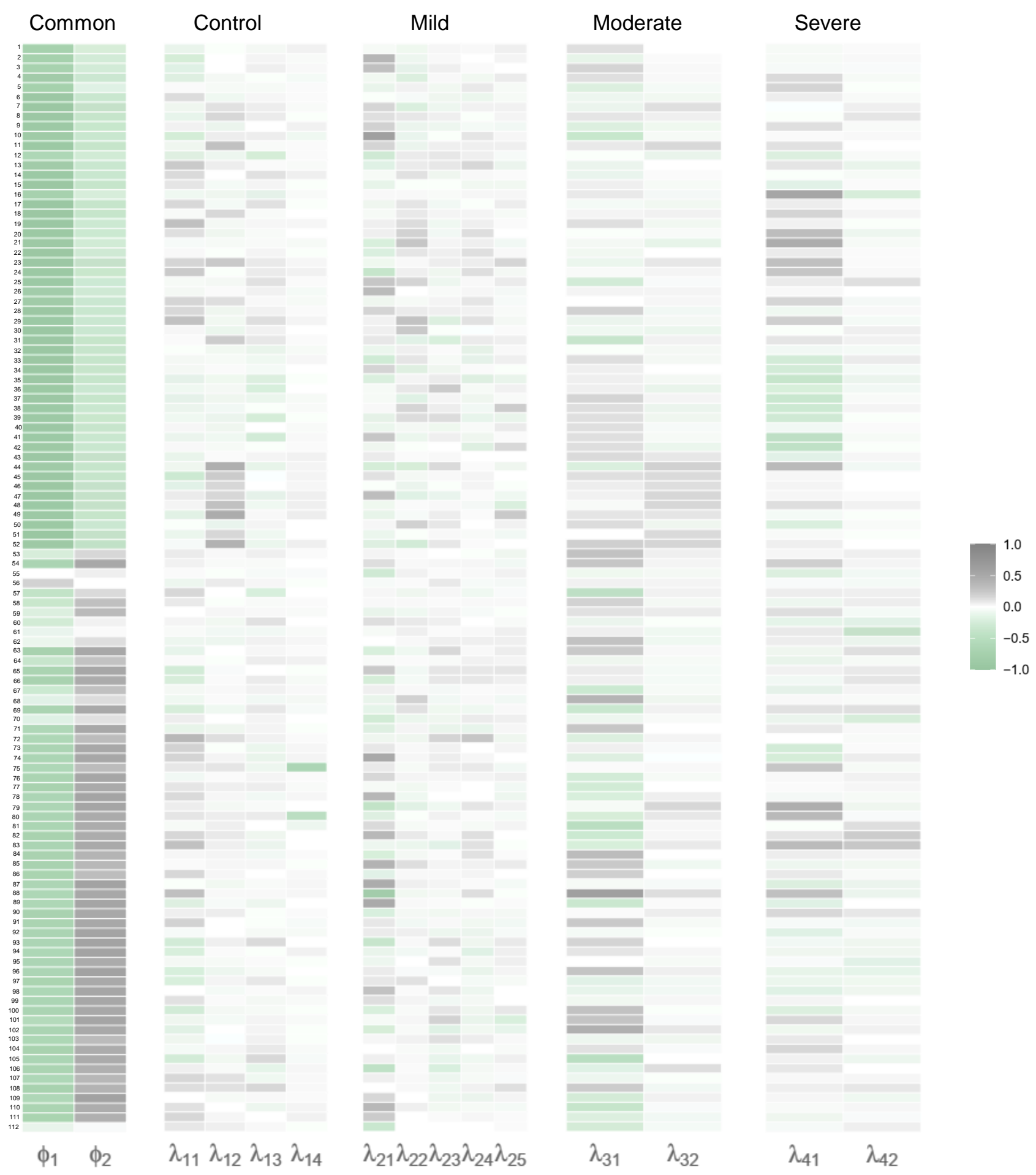


a
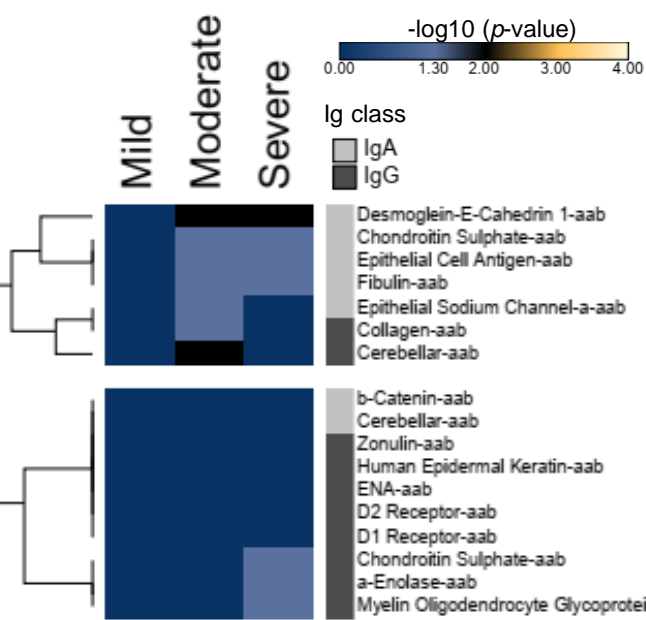

Desmoglein-E-Cahedrin 1-aa Epithelial Cell Antigen-aab Fibulin-aab

Epithelial Sodium Channel-a-aab Collagen-aab

\section{Cerebellar-aab}

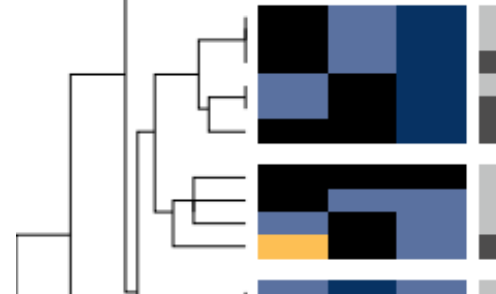

b-Catenin-aab

Cerebellar-aab

Zonulin-aab

Human Epidermal Keratin-aab

ENA-aab

D2 Receptor-aab

D1 Receptor-aab

Chondroitin Sulphate-aab

Myelin Oligodend

2 Receptor-aab

Myelin Basic Protein-aab

b-Catenin-aab

Acetycholine Receptor-aab

Amyloid-b Peptide-aab

Enteric Nerve-aab

Transglutaminase 2-aab

Troceptor-aab

Tau Protein-azb

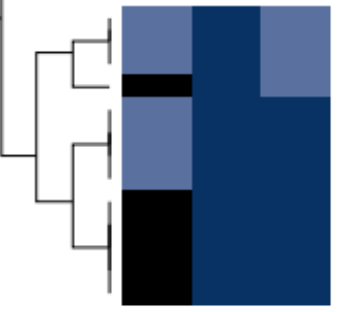

Claudin 7.2-aab

Heparin-aab

Islet Cell Antigen-aab

Insulin Receptor-aab

Neurofilament Proteins-aab

Claudin 7.1-aab

Somatotropin-aab

Fibrinogen-aab

Lung Surfactant Protein-C-aab

Transglutaminase 6-aab

Ganglioside-aab

Fibulin-aab
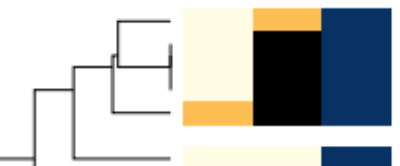

a-Synucleins-aab

Amyloid-b Peptide-aab

Liver Microsomal Antigen-aab

GAD65-aab

Insulin Receptor-aab

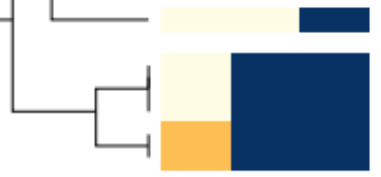

Transglutaminase 3-aab

Human Epidermal Keratin-aab

Transglutaminase 2-aab

Insulin-aab

Epithelial Cell Antigen-aab

Sulphatide-aab

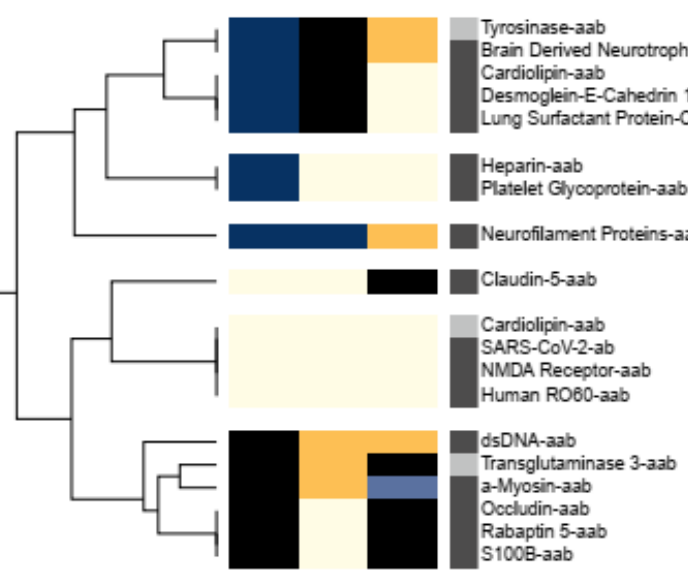

e

group $\mathrm{E}$ Control Mild $\mathrm{E}$ Moderate $\mathrm{E}$ Severe

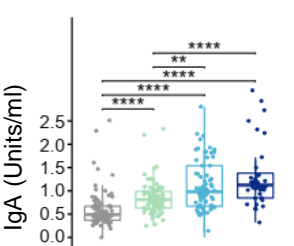

Cardiolipin-aab

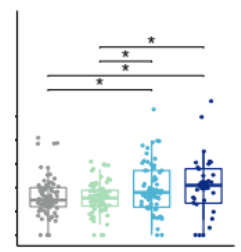

CS-aab

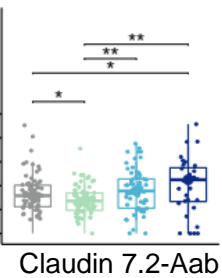

Claudin 7.2-Aab b

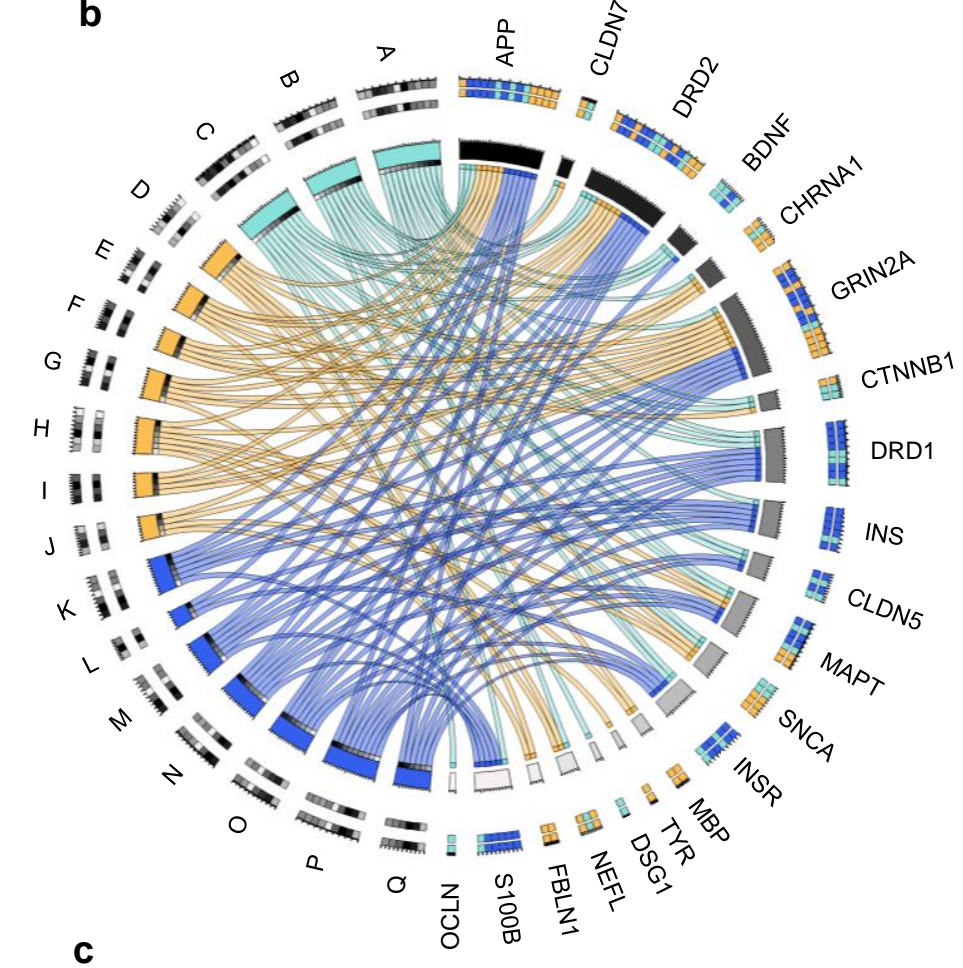

cell junction assembly

negative regulation of synaptic transmission dopamine metabolic process

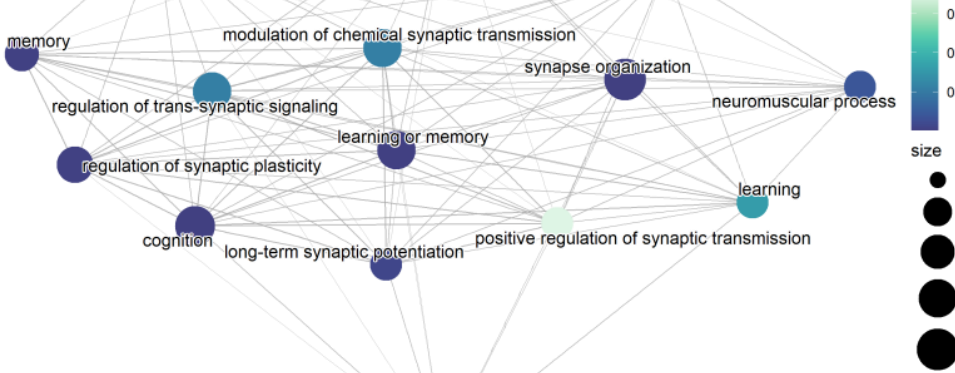

d

neuron projection maintenance

group Control $_{\text {Mild }}$ Moderate
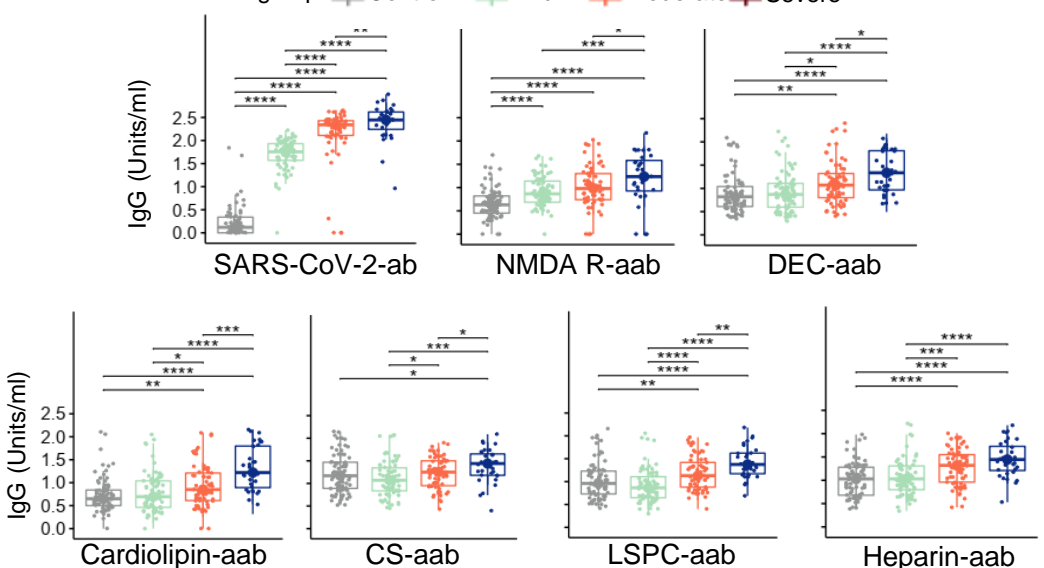

Heparin-aab

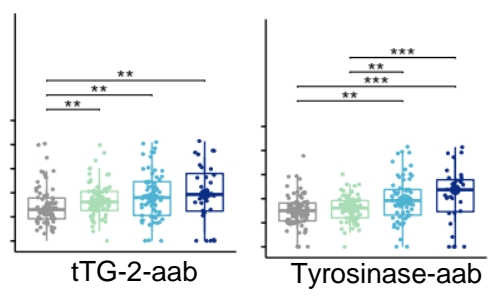



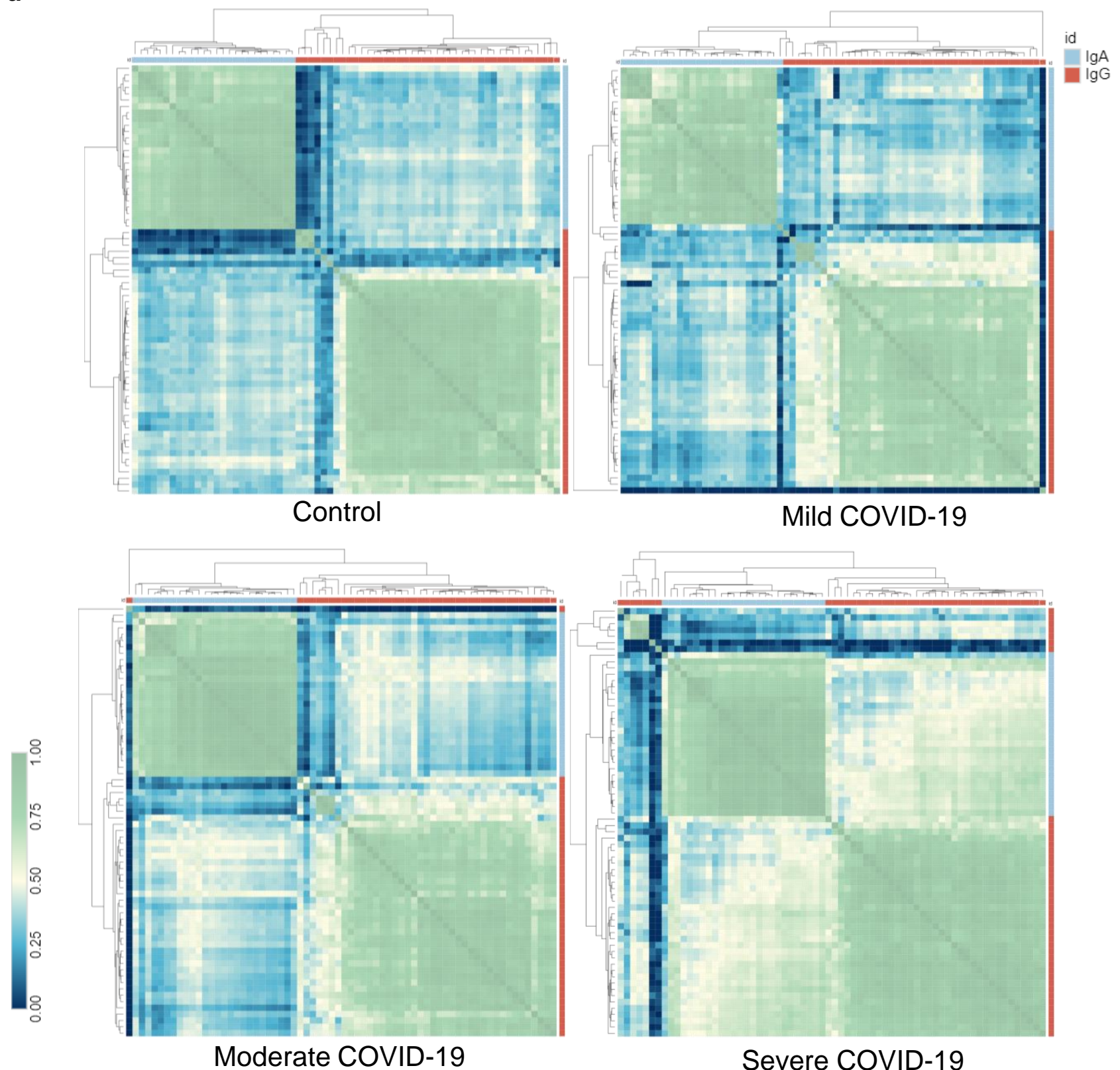

b

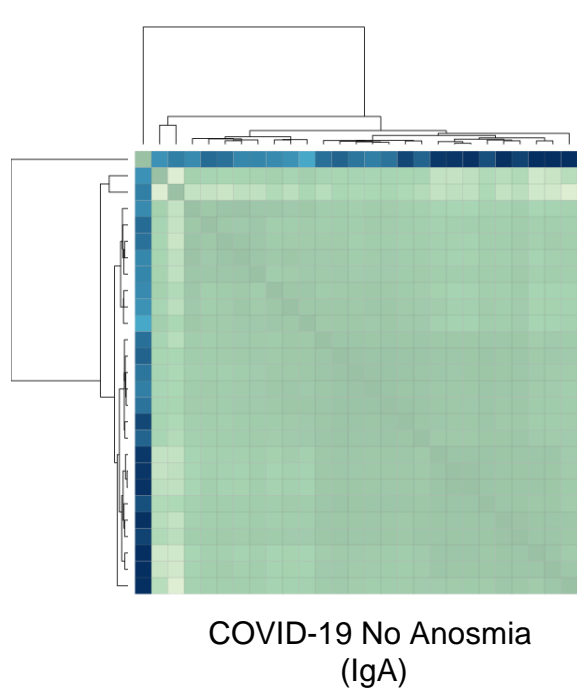

Severe COVID-19

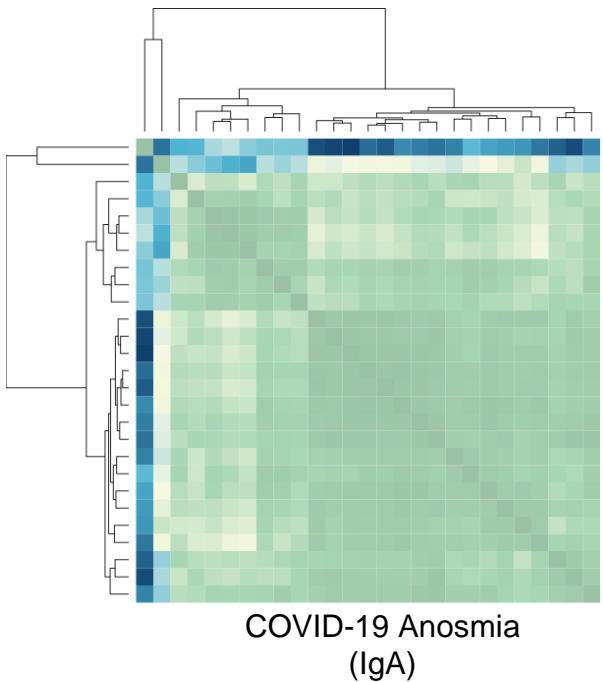

$(\lg A)$

$(\lg A)$

$(\lg \mathrm{A})$ 
a

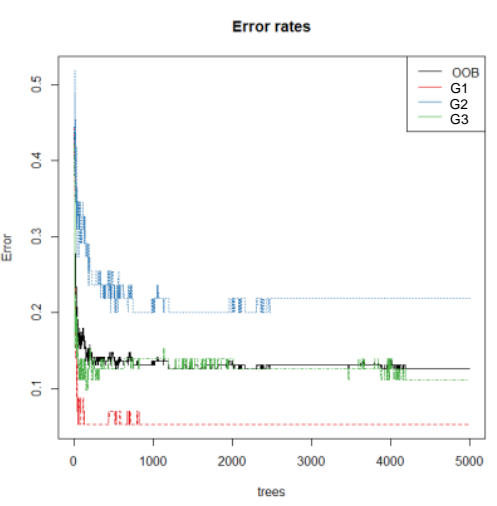

d

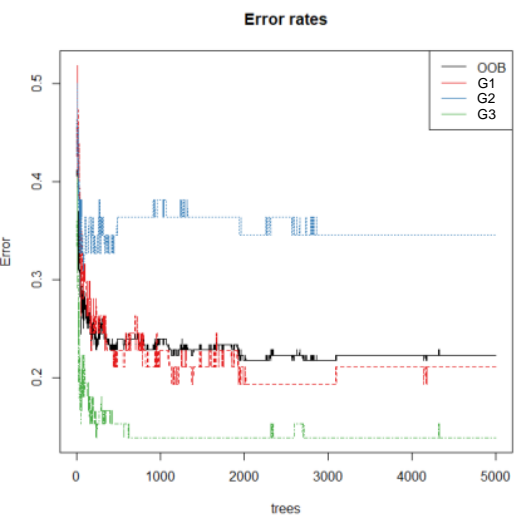

b

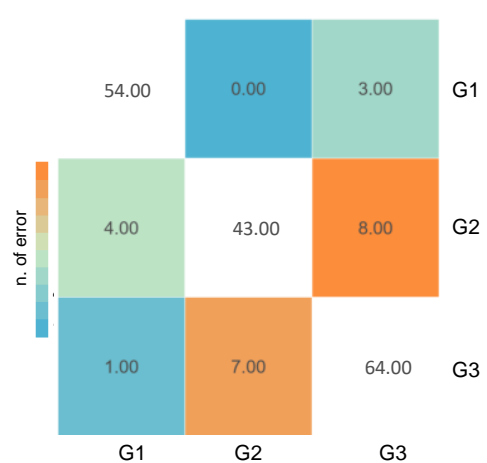

e

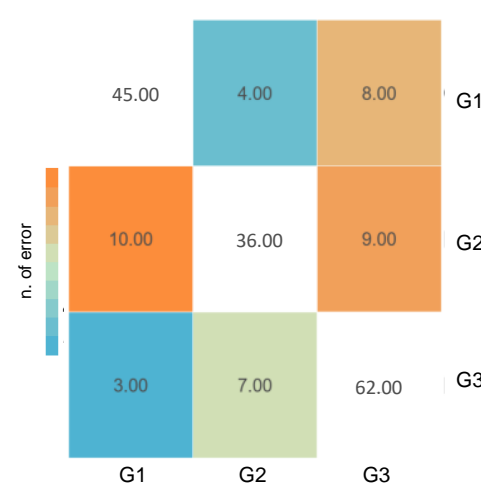

C

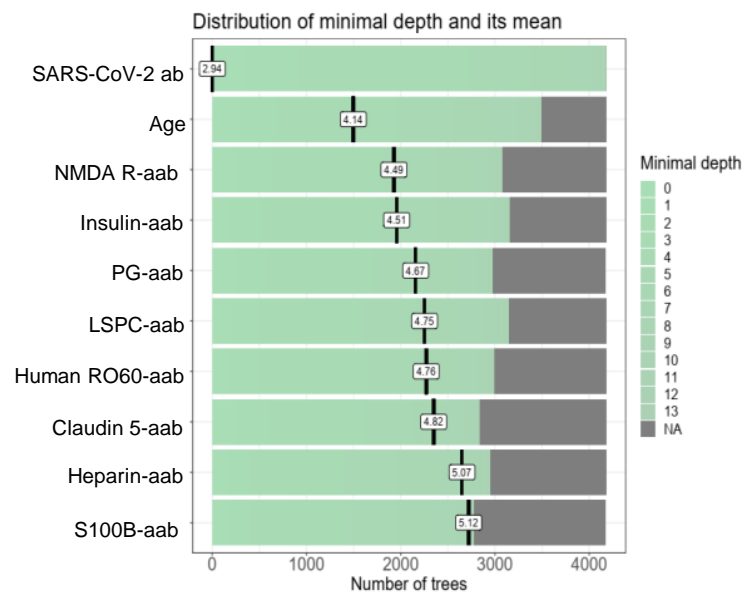

f

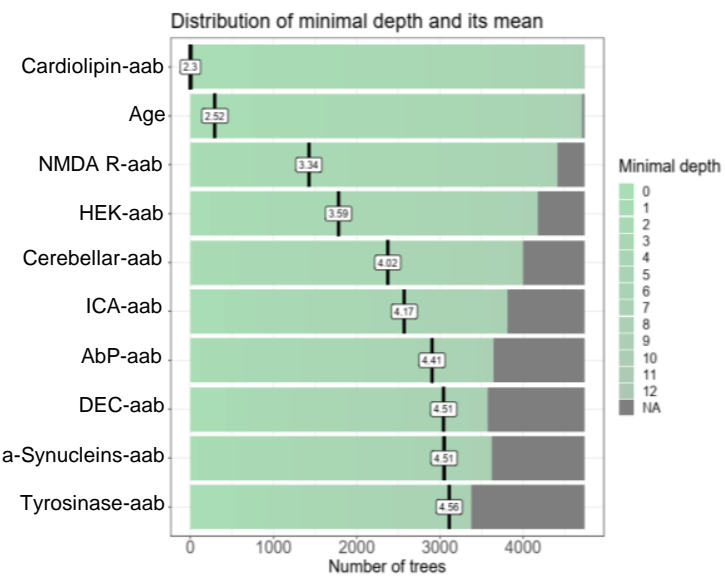

h

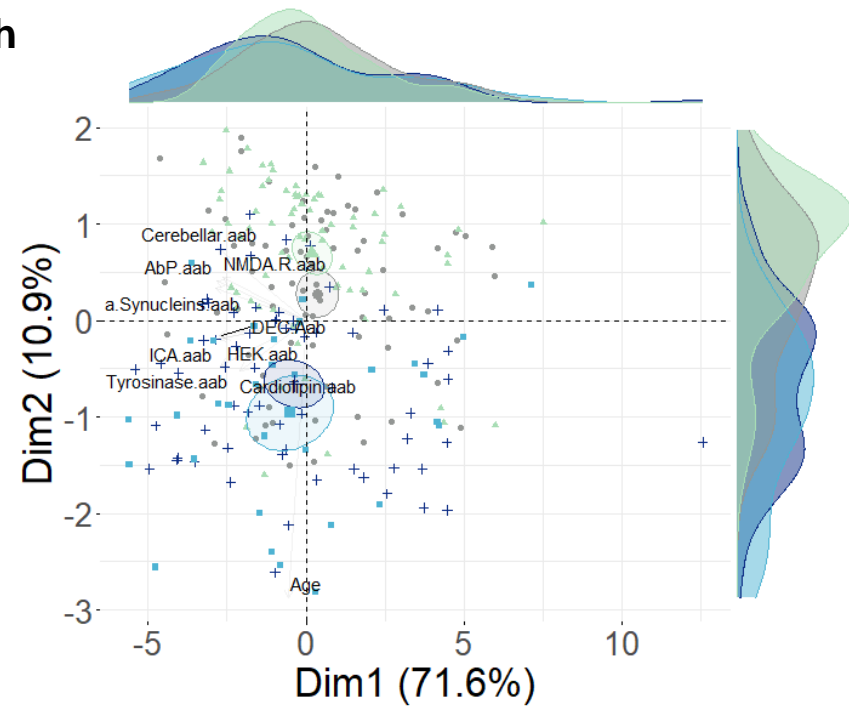

- Control $\triangle$ Mild $\square$ Moderate + Severe 
a

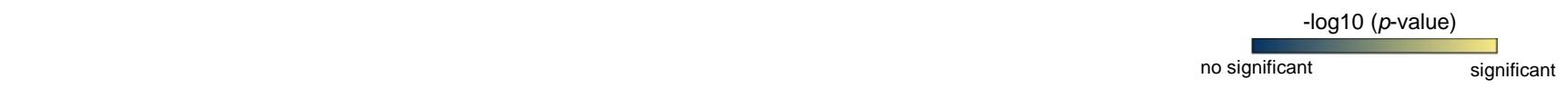

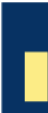

1

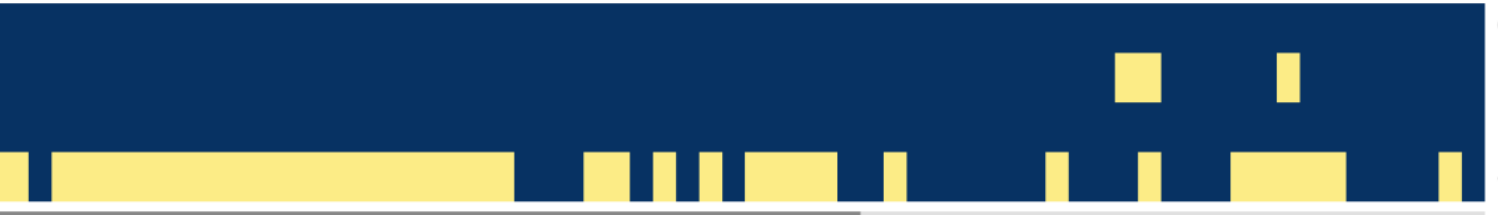

Control

Mild

Moderate

Ig class

Severe

$\lg A$
$\lg G$
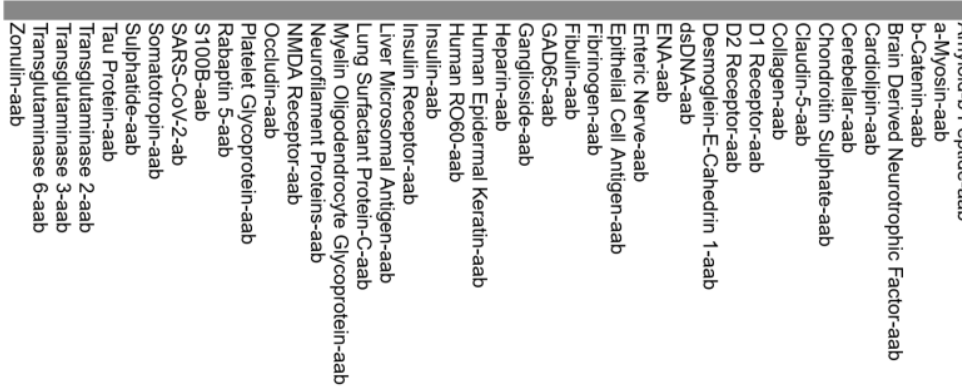

b
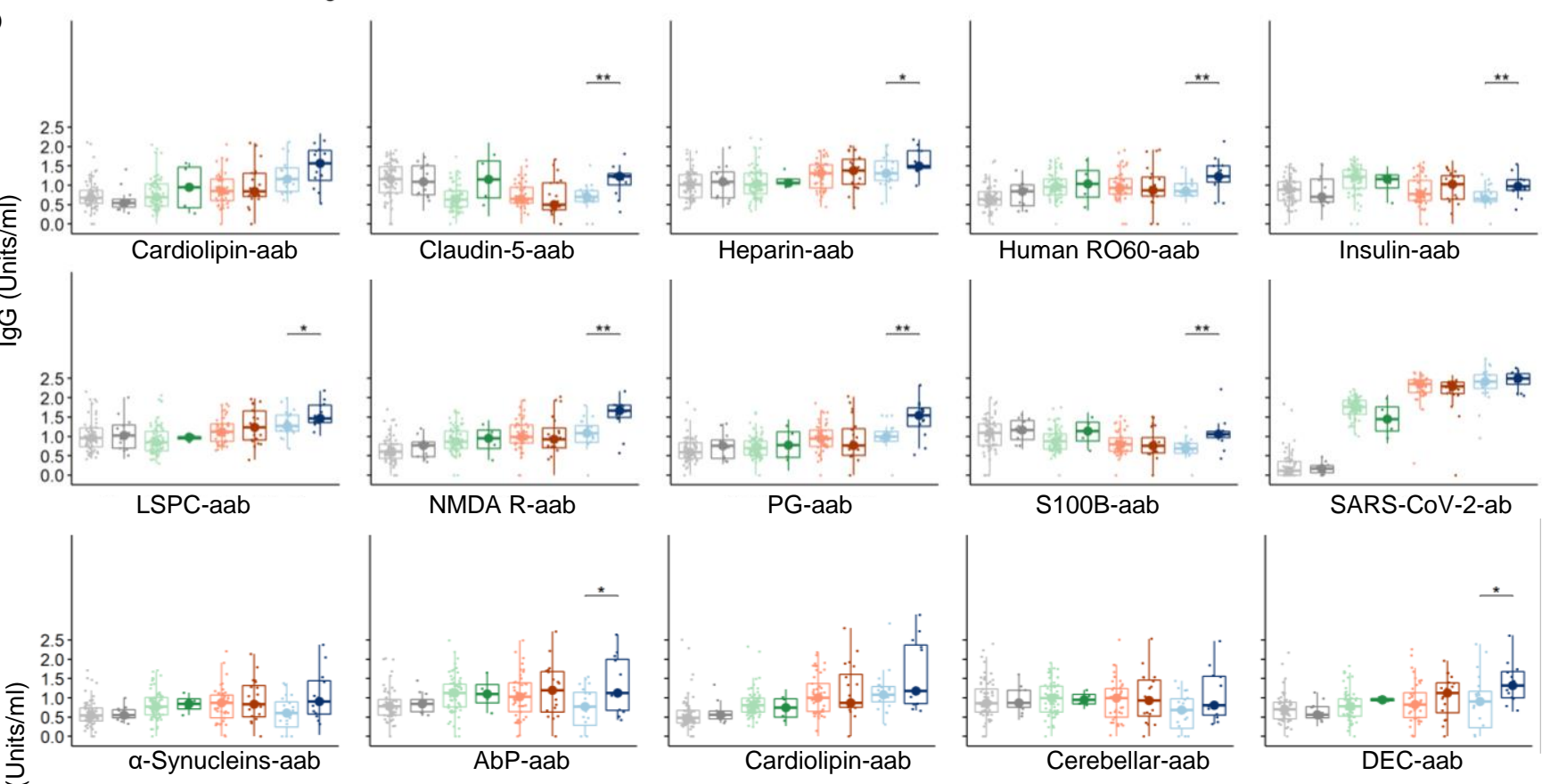

Human RO60-aab
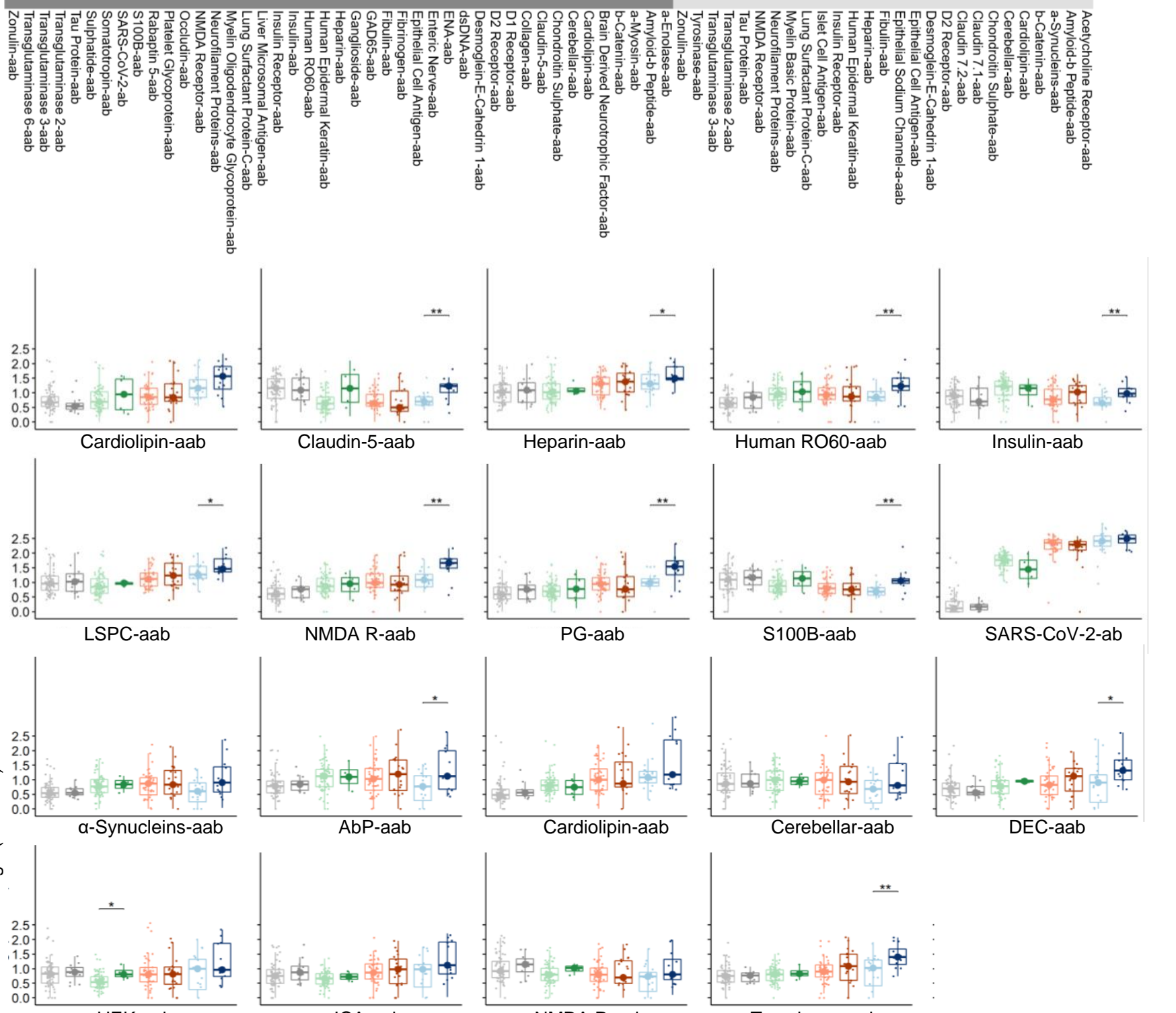

HEK-aab

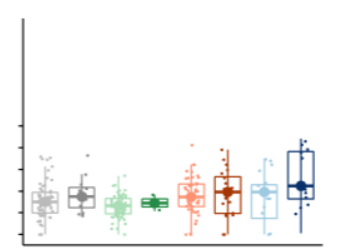

ICA-aab
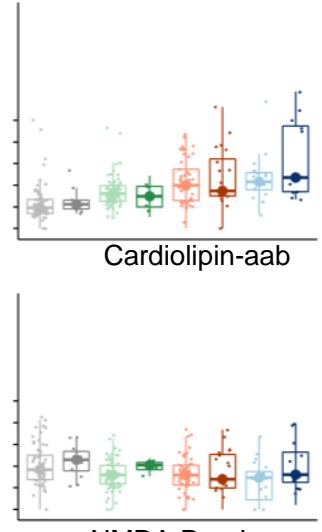

NMDA R-aab
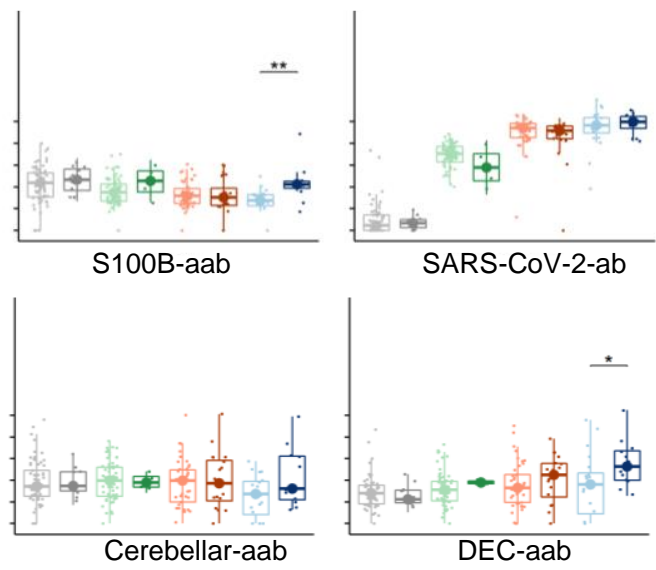

*
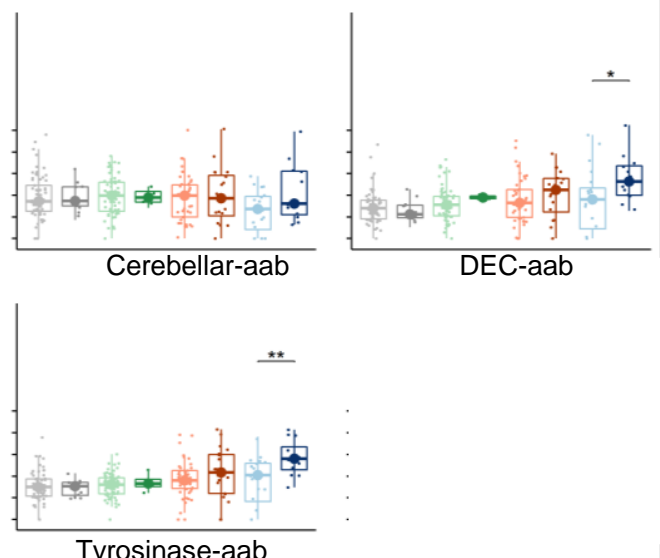


\section{FIGURES} It is made available under a CC-BY-NC-ND 4.0 International license.

Figure 1. Study workflow. a) Schematic overview of study cohorts, statistics and bioinformatics analyses carried out to characterize the spectrum of autoantibodies linked to diverse autoimmune diseases that associate with COVID-19 outcomes.

Figure 2. Perturbation of normal autoantibody settings in COVID-19. Multi-study factor analysis (MSFA) of all IgA and IgG autoantibodies as well as anti-SARS-CoV-2 IgG antibodies that were analyzed. The heatmaps show estimated factor loadings of common $(\phi)$ and specific $(\lambda)$ latent factors of each group. Positive and negative loadings range from -1 (green) to 1 (grey), respectively. Autoantibody number shown according to their names in Supplementary Table 1. See Supplementary Table 6 for the loadings.

Figure 3. Autoantibodies and functional association of their targets. a) Heatmap showing the hierarchical cluster of $\operatorname{IgG}$ and $\operatorname{IgA}$ autoantibodies significantly different when comparing each COVID-19 cohort (mild, moderate, and severe groups) with healthy controls. The bar, ranging from blue to black and light yellow, shows the range of significance from - $\log 10(p$ value $)=0$ to 4 . Rows were clustered using Euclidean distance between $-\log 10$ ( $p$-values). Ig, immunoglobulin. b) The barplots exhibt the number of $\operatorname{IgG}$ and $\operatorname{IgA}$ autoantibodies that were evaluated and found as significant or not. c) Network of biological processes (BP) enriched (adjusted p-value $<0.05$ ) by the autoantibody targets. The edges connect related BPs. The circles' colors and size correspond to the adjusted $p$-value and number of autoantibody targets enriching BPs, respectively. d) Circos plot illustrates the top 10 BPs enriched by both IgA and IgG autoantibody targets. Suplementary Table 4 and Supplementary Fig. 3 show all gene ontology (GO) categories (BPs and signaling pathways) enriched by the autoantibody targets. For this analysis, we selected autoantibody targets from significantly different autoantibodies among the groups. BPs are denoted by letters A-Q (A: synapse organization; B: learning or memory; C: cell junction assembly; D: positive regulation of peptidase activity; E: response to alcohol; F: excitatory postsynaptic potential; G: neuromuscular process; H: positive regulation of proteolysis; I: chemical synaptic transmission, postsynaptic; J: phenol-containing compound metabolic process; K: long-term synaptic potentiation; L: neuron projection maintenance; $\mathrm{M}$ : positive regulation of synaptic transmission; $\mathrm{N}$ : regulation of synaptic plasticity; $\mathrm{O}$ : memory; $\mathrm{P}$ : cognition; Q: learning). The blue, yellow, and green colors correspond to $\operatorname{IgA}, \operatorname{IgG}$, and shared terms, respectively. Box plots exemplifying d) $\operatorname{IgG}$ and e) $\operatorname{IgA}$ autoantibodies significantly ( different between healthy controls and COVID-19 groups. See Supplementary Table 2 for full names and abbreviations of autoantibodies and their targets. All autoantibodies with significant differences between at least two groups are shown in Supplementary Figures 1 and 2. Each box plot shows the median with first and third interquartile range (IQR), whiskers representing minimum and maximum values within IQR, and individual data points. Significance was determined using two-sided Wilcoxon rank-sum tests and is indicated by asterisks $(* \mathrm{p} \leq 0.05$, $* * \mathrm{p} \leq 0.01, * * * \mathrm{p} \leq 0.001$, and $* * * * \mathrm{p} \leq 0.0001)$. Control $(n=77)$; mild $(n=74)$, moderate $(n$ $=65)$, and severe $(n=32)$ COVID-19.

Figure 4. Hierarchical clustering of the autoantibody correlation signatures according to disease severity and anosmia. Heatmap of significant different aab vs. aab correlations: a) IgG and IgA autoantibodies in healthy control and COVID-19 groups and b) IgA autoantibodies of COVID-19 patients with or without anosmia versus healthy controls. See Supplementary Fig. 4a-4d for correlograms with the autoantibody names; Supplementary Fig. 4e for IgG autoantibody correlations in patients with anosmia versus no anosmia; and Supplementary Fig. 5 a-b for IgG and IgA autoantibodiy correlations for healthy controls and COVID-19 groups. The color scale bar ranging from 0 to 1 represents Spearman's rank correlation coefficient. Rows and columns were clustered based on Euclidean distance. See Supplementary Table 5 
medRxiv preprint doi: https://doi.org/10.1101/2022.02.17.22271057; this version posted February 18, 2022. The copyright holder for this preprint (which was not certified by peer review) is the author/funder, who has granted medRxiv a license to display the preprint in perpetuity. for correlations matrices with the correlation coefficient values. Control int $(n=77)$; mild $(n=74)$, moderate $(n=65)$, and severe $(n=32)$ COVID-19. No anosmia $(n=69)$ and anosmia $(n=101)$.

Figure 5. Ranking autoantibodies as predictors of COVID-19 severity. Stable curve showing number of trees and error rates for a) IgG and d) IgA autoantibodies with out-of-bag (OOB) estimate of $12,5 \%$ and $22.28 \%$ error rate, respectively. Heatmap presents the confusion matrix of b) $\operatorname{IgG}$ and e) $\operatorname{IgA}$ autoantibodies, the number of individuals classification by group (healthy controls $=\mathrm{G} 1$; mild $=\mathrm{G} 2$; moderate+severe $=\mathrm{G} 3$ ). The color scale ranging from blue to orange corresponds to the number of errors or hits by groups denoted inside the colored or white squares, respectively. Control $(n=77)$; mild $(n=74)$, moderate+severe $(n=97)$ COVID19. Bar plots of the top 10 strongest c) IgG (also anti-SARS-CoV-2 IgG antibodies and age) and f) IgA autoantibodies predictors of COVID-19 patient classification according to disease severity. The variables are shown according to minimal depth and number of trees. The color scale bar ranging from 0 to 13 represents the minimal and maximum minimal depth. The small dark vertical bars represent the mean of minimal depth for each variable. Principal component analysis (PCA) with spectral decomposition shows that g) $\operatorname{IgG}$ and h) $\operatorname{IgA}$ autoantibodies stratify COVID-19 patients according to disease severity, revealing the overlap between the moderate and severe cohorts. The PCA was carried out based on the top 10 most important variables as listed in $\mathbf{c}$ and $\mathbf{f}$, respectively. Control $(n=77)$; mild $(n=74)$, moderate $(n=65)$, and severe $(n=32)$ COVID-19. See Supplementary Table 2 for full names and abbreviations of autoantibodies and their targets.

Figure 6. Age-dependent elevation of autoantibody levels in patients with severe SARSCoV-2 infection. a) Heatmap showing the hierarchical cluster of IgG and IgA autoantibodies significantly different when comparing young versus elderly controls or young versus elderly of each COVID-19 cohort. The colors blue and light yellow indicate autoantibodies with or without significant differences between the young versus elderly in each group. b) Box plots display changes in the autoantibody levels by age (young $<60$ and elderly $\geq 60$ ). Each box plot shows the median with first and third interquartile range (IQR), whiskers representing minimum and maximum values within IQR, and individual data points. Significance was determined using two-sided Wilcoxon rank-sum tests and is indicated by asterisks $\left(* p \leq 0.05,{ }^{* *} p \leq 0.01\right.$, $* * * \mathrm{p} \leq 0.001$, and $* * * * \mathrm{p} \leq 0.0001)$. control-young $(n=65)$ and control-elderly $(n=12)$; mildyoung $(n=70)$, mild-elderly $(n=4)$, moderate-young $(n=45)$, moderate-elderly $(n=20)$, severe-young $(n=20)$, severe-elderly $(n=12)$ COVID-19 patients. All autoantibodies with significant differences between at least two groups are shown in Supplementary Fig. 7. 